\title{
ディスクリプタ表現を利用した倒立振子システムの非線形 $\mathcal{H}_{\infty}$ 制御
}

\author{
川田昌 克*・井上 和 夫**
}

Nonlinear $\mathcal{H}_{\infty}$ Control for an Inverted Pendulum System Using Descriptor Representation ${ }^{\dagger}$

Masakatsu KaWATA* and Kazuo Inoue**

\begin{abstract}
In this paper, a design method of the nonlinear $\mathcal{H}_{\infty}$ state feedback is proposed for a nonlinear descriptor model of an inverted pendulum system. First, the nonlinear descriptor model of a polynominal type with structural uncertainties for the inverted pendulum system introduced. The descriptor variables of this model include not only state variables but also the time derivative of state variables. Next, a state-dependent matrix inequality condition, which is can be transferred to the finite number of linear matrix inequalities, for the nonlinear robust $\mathcal{H}_{\infty}$ control problem and the eigenvalue location problem is given. The validity of the proposed nonlinear $\mathcal{H}_{\infty}$ control is shown through experiments as compared with the other nonlinear $\mathcal{H}_{\infty}$ control.
\end{abstract}

Key Words: nonlinear $\mathcal{H}_{\infty}$ control, eigenvalue location, descriptor form, linear matrix inequality, inverted pendulum

\section{1.はじめに}

ディスクリプタ方程式は多くのシステムでその物理構造を 適切に表すことができるため，これまでにディスクリプタシ ステムに対する各種制御問題が考察されている，従来，ディ スクリプタ変数を状態変数 $\boldsymbol{x}$ とし,$\dot{x}$ の係数行列の正則性 を仮定することが多かったが1) 3)，近年，ディスクリプ夕形 式の柔軟性を利用したゲインスケジュールド $\mathcal{H}_{\infty}$ 制御 ${ }^{4)}, 2$ 次安定化制御 5), 6), コスト保証制御 ${ }^{7)}$ などの設計法が提案さ れている。これら設計法では，たとえばスケジューリングパ ラメータ (あるいは不確かなパラメータ) $\boldsymbol{p}$ に依存した

$$
\boldsymbol{E}(p) \dot{\boldsymbol{x}}=\boldsymbol{A}(\boldsymbol{p}) \boldsymbol{x}+\boldsymbol{B u}
$$

が与えられたとき $(\boldsymbol{E}(\boldsymbol{p}), \boldsymbol{A}(\boldsymbol{p})$ は $\boldsymbol{p}$ に関して有限のべき級 数), ディスクリプタ形式の柔軟性を利用して

$$
\boldsymbol{E}^{\sharp} \dot{\boldsymbol{\xi}}=\boldsymbol{A}^{\sharp}(\boldsymbol{p}) \boldsymbol{\xi}+\boldsymbol{B}^{\sharp} \boldsymbol{u}
$$

のように変換することにより, $\boldsymbol{p}$ をディスクリプタ変数 $\boldsymbol{\xi}$ の 係数行列 $\boldsymbol{A}^{\sharp}(\boldsymbol{p})$ に集約しており，保守性の軽減に有効であ る。このとき，一般に $\boldsymbol{E}^{\sharp}$ は非正則となるが，増淵ら ${ }^{8)}$,9) や 上里ら ${ }^{6)}$ が示した結果を利用すれば，可解条件として線形行

$†$ 第 28 回制御理論シンポジウムで発表 $(1999 \cdot 5)$

* 舞鶴工業高等専門学校電子制御工学科 舞鶴市字白屋 234

** 立命館大学理工学部 草津市野路東 1-1-1

* Department of Control Engineering, Maizuru National College of Technology, Maizuru

** Faculty of Science and Engineering, Ritsumeikan University, Kusatsu

(Received January 11, 1999)

(Revised March 29, 2000)
列不等式 (LMI; linear matrix inequality) が得られる. 特 に，上里らの結果を用いると $\boldsymbol{E}^{\sharp}$ の構造に依存せずに等式制 約のない LMI 条件となり，既存のソフトウェアを用いて容 易に解が得られるため沉用性が高い.

本論文で対象とする倒立振子システムの数学モデルは，非 線形ディスクリプタ方程式で適切に表すことができ，ディス クリプタ形式を利用した設計法が有効であると考えられる。 そこで，本論文では，ディスクリプ夕表現を利用した非線形 $\mathcal{H}_{\infty}$ 状態フィードバックを設計し, 実機実験によりその有効 性を検証することを目的とする。

本論文では，まず，倒立振子システムの非線形ディスクリ プタ方程式における係数行列が状態变数 $\boldsymbol{x}$ に関して有限のべ き級数で表されていないため, 係数行列を $x=\mathbf{o}$ で Taylor 級数展開し, 状態変数 $\boldsymbol{x}$ に関して有限のべき級数と構造的不 確かさの和で表す。つきに，構造的不確かさを考慮したゲイ ンスケジューリング 10),11)の考え方に基づき，ディスクリプ 夕変数を $\boldsymbol{x}$ のみとしたディスクリプタ形式の一般化制御対 象を構成し, 非線形 $\mathcal{H}_{\infty}$ 制御問題の可解条件を状態に依存 した線形行列不等式 (sLMI; state-dependent linear matlix inequality) で表すことを提案する ${ }^{12)}$. 得られる sLMI 条件 は有限個の LMI に帰着可能であるため, 既存のソフトウェ アを利用して容易に解を得ることができ，解析解を求めるこ とが困難な Hamilton-Jacobi 方程式 (不等式)に基づく設計 法 $\left.{ }^{13)}, 14\right)$ よりも実用的である。また，非線形 $\mathcal{H}_{\infty}$ 制御問題 のみを考えて設計すると, 状態フィードバックゲインが過大 となる傾向があるが，線形システムの極配置問題 ${ }^{2)} に$ 相当す る固有値指定問題も制御仕様として考えることによって，こ の問題点を回避できる。しかしながら，倒立振子システムの 
場合，ディスクリプタ変数 $\boldsymbol{x}$ の時間微分 $\dot{x}$ の係数行列が $x$ に依存しているため, SLMI を有限個の LMI で置き換えた ときに生じる保守性や評価出力における構造的不確かさを見 積もる際の保守性に関する対処が十分ではない ${ }^{15)}$.

そこで,つぎに, ディスクリプタ形式の柔軟性を積極的に 利用し, 状態変数 $x$ だけでなくその時間微分 $\dot{x}$ もディスク リプ夕変数に含め ${ }^{4)}$, 保守性を軽減することを提案する ${ }^{15)}$. その際, 上里らの結果を用いても円内への固有值指定問題の 可解条件に現れる等式制約を取り除くことができないため, この問題点の対処法について議論する. また, 非線形 $\mathcal{H}_{\infty}$ 制 御問題と固有値指定問題の複数仕様をできるだけ保守性なく 満足させるための SLMI の解の構造についても考察する.

最後に, 実機実験により Hamilton-Jacobi 方程式 (不等 式)の近似解に基づく設計法 ${ }^{16)}$,17) との比較を行い, 本論文 で提案する設計法の有効性を示す.

以下に本論文で用いる記号の説明を行う ${ }^{14)}$. 正方行列 $\boldsymbol{A}$ に対して $\mathrm{He}[\boldsymbol{A}]:=\boldsymbol{A}+\boldsymbol{A}^{T}$ と定義し, 対称行列を

$$
\left[\begin{array}{cc}
\boldsymbol{A} & \boldsymbol{B} \\
\boldsymbol{B}^{T} & \boldsymbol{C}
\end{array}\right]=\left[\begin{array}{cc}
\boldsymbol{A} & \star \\
\boldsymbol{B}^{T} & \boldsymbol{C}
\end{array}\right]=\left[\begin{array}{cc}
\boldsymbol{A} & \boldsymbol{B} \\
\star & \boldsymbol{C}
\end{array}\right]
$$

のように表す. また, $\boldsymbol{x} \in \mathcal{R}^{n}$ が $\int_{t_{0}}^{\infty}\|\boldsymbol{x}\|^{2} d t<\infty$ であると き $x \in \mathcal{L}_{2}$ と表し,$x$ の $\mathcal{L}_{2}$ ノルムは $x \in \mathcal{L}_{2}$ に対して

$$
\|x\|_{2}=\left(\int_{t_{0}}^{\infty}\|x\|^{2} d t\right)^{1 / 2}
$$

と定義される. 有界な関数全体を $\mathcal{L}_{\infty}$ と表し, 特に $\sup _{t}\|x\| \leq$ $c$ ( $c$ は正定数 $)$ であるとき, $\boldsymbol{x} \in \mathcal{L}_{\infty}^{c}$ と表す.

\section{2. 問題設定}

\section{1 倒立振子システムの数学モデル}

Fig. 1 に示す倒立振子システムの数学モデルは,

$$
\begin{aligned}
\bar{M} \ddot{r}+(m \ell \cos \theta) \ddot{\theta} & =-\mu_{1} \dot{r}+m \ell \dot{\theta}^{2} \sin \theta+k_{f} v \\
(m \ell \cos \theta) \ddot{r}+\bar{J} \ddot{\theta} & =-\mu_{2} \dot{\theta}+m \ell g \sin \theta
\end{aligned}
$$

で与えられる。たたし， $\bar{M}=M+m, \bar{J}=J+m \ell^{2}$ で あり, $r[\mathrm{~m}]$ : 台車の変位, $\theta[\mathrm{rad}]$ : 振子の角度, $v[\mathrm{~V}]$ : ドライバに加える電圧, $M=0.338[\mathrm{~kg}]$ : 台車の質量, $m=0.102[\mathrm{~kg}]$ : 振子の質量, $\ell=0.158[\mathrm{~m}]$ : 軸から振 子の重心までの距離, $J=1.76 \times 10^{-3}\left[\mathrm{~kg} \cdot \mathrm{m}^{2}\right]$ : 振子の 重心まわりの慣性モーメント, $\mu_{1}=5.92[\mathrm{~kg} / \mathrm{s}]$ : 台車駆

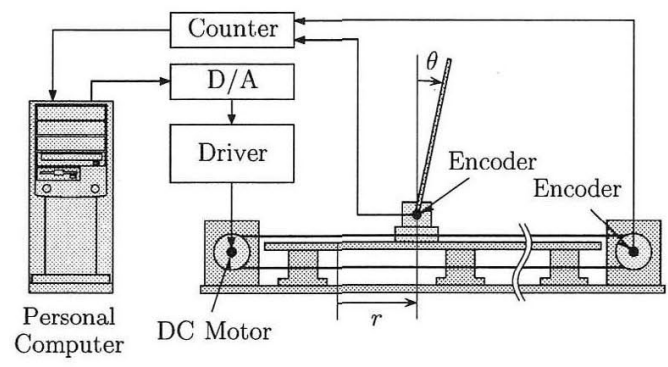

Fig. 1 Inverted pendulum system
動系の等価粘性摩擦係数, $\mu_{2}=3.86 \times 10^{-4}\left[\mathrm{~kg} \cdot \mathrm{m}^{2} / \mathrm{s}\right]$ : 軸の粘性摩擦係数, $k_{f}=3.07[\mathrm{~N} / \mathrm{V}]$ : 力 $/$ 電圧変換係数, $g=9.81\left[\mathrm{~m} / \mathrm{s}^{2}\right]$ : 重力加速度を表す. したがって, 状態変数 を $\boldsymbol{x}_{p}:=\left[\begin{array}{cccc}r & \dot{r} & \theta & \dot{\theta}\end{array}\right]^{T}$, 操作量を $u_{p}:=v$ とすると, システムの構造を適切に表した以下の非線形ディスクリプタ 方程式が得られる ${ }^{12)}$.

$$
\begin{aligned}
& \mathcal{P}: \boldsymbol{E}_{p}\left(x_{p}\right) \dot{\boldsymbol{x}}_{p}=\boldsymbol{A}_{p}\left(\boldsymbol{x}_{p}\right) \boldsymbol{x}_{p}+\boldsymbol{B}_{p} u_{p} \\
& \boldsymbol{E}_{p}\left(\boldsymbol{x}_{p}\right)=\left[\begin{array}{cccc}
1 & 0 & 0 & 0 \\
0 & \bar{M} & 0 & m \ell \cos \theta \\
0 & 0 & 1 & 0 \\
0 & m \ell \cos \theta & 0 & \bar{J}
\end{array}\right] \\
& \boldsymbol{A}_{p}\left(\boldsymbol{x}_{p}\right)=\left[\begin{array}{cccc}
0 & 1 & 0 & 0 \\
0 & -\mu_{1} & m \ell \dot{\theta}^{2} \frac{\sin \theta}{\theta} & 0 \\
0 & 0 & 0 & 1 \\
0 & 0 & m \ell g \frac{\sin \theta}{\theta} & -\mu_{2}
\end{array}\right] \\
& \boldsymbol{B}_{p}=\left[\begin{array}{lccc}
0 & k_{f} & 0 & 0
\end{array}\right]^{T}
\end{aligned}
$$

\section{2 一般化制御対象の構成}

倒立振子システムの非線形サーボ制御を実現するため，

Fig. 2 に示す拡大系を考える ${ }^{17)}$ 。たたし，重み関数は

$$
W_{t}(p)=b_{t 0}+b_{t 1} p+b_{t 2} p^{2}, W_{s}(p)=\frac{b_{s}}{p}
$$

であり, $p:=d / d t$ は微分演算子である. $r^{r e f}$ を $r$ の目標値 とすると, 評価出力 $\bar{z}:=\left[\begin{array}{ll}\bar{z}_{1} & \bar{z}_{2}\end{array}\right]^{T}$ は

$$
\begin{aligned}
\bar{z}_{1} & =W_{t}(p) r=C_{t 0} \boldsymbol{x}_{p}+\boldsymbol{C}_{t 1} \dot{\boldsymbol{x}}_{p} \\
\bar{z}_{2} & =W_{s}(p)\left(r^{r e f}-r\right)=b_{s} x_{s} \\
\boldsymbol{C}_{t 0} & =\left[\begin{array}{llll}
b_{t 0} & b_{t 1} & 0 & 0
\end{array}\right], \boldsymbol{C}_{t 1}=\left[\begin{array}{llll}
0 & b_{t 2} & 0 & 0
\end{array}\right] \\
x_{s} & =\int_{0}^{t}\left(r^{r e f}-C_{p 1} x_{p}\right) d t, C_{p 1}=\left[\begin{array}{llll}
1 & 0 & 0 & 0
\end{array}\right]
\end{aligned}
$$

となるから, 状態変数, 操作量, 外生信号をそれぞれ $x:=$ $\left[\begin{array}{lll}x_{1} & \cdots & x_{5}\end{array}\right]^{T}=\left[\begin{array}{ll}x_{p}^{T} & x_{s}\end{array}\right]^{T}, u:=u_{p}, \bar{w}:=r^{r e f}$ と定義すると, 一般化制御対象は次式で与えられる。

$$
\begin{gathered}
\overline{\mathcal{S}}:\left\{\begin{array}{l}
\overline{\boldsymbol{E}}_{N}(\boldsymbol{x}) \dot{\boldsymbol{x}}=\overline{\boldsymbol{A}}_{N}(\boldsymbol{x}) \boldsymbol{x}+\overline{\boldsymbol{B}}_{1} \bar{w}+\overline{\boldsymbol{B}}_{2} u \\
\overline{\boldsymbol{z}}=\overline{\boldsymbol{C}}_{10} \boldsymbol{x}+\overline{\boldsymbol{C}}_{11} \dot{\boldsymbol{x}} \\
\boldsymbol{y}=\boldsymbol{x}
\end{array}\right. \\
\overline{\boldsymbol{E}}_{N}(\boldsymbol{x})=\left[\begin{array}{cc}
\boldsymbol{E}_{p}\left(\boldsymbol{x}_{p}\right) & \mathbf{o} \\
\mathbf{o} & 1
\end{array}\right]
\end{gathered}
$$

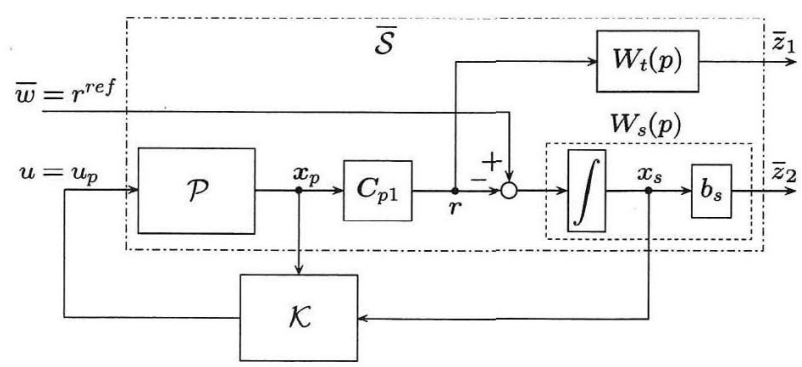

Fig. 2 Nonlinear servo system 


$$
\begin{aligned}
& \overline{\boldsymbol{A}}_{N}(\boldsymbol{x})=\left[\begin{array}{cc}
\boldsymbol{A}_{p}\left(\boldsymbol{x}_{p}\right) & \mathbf{0} \\
-\boldsymbol{C}_{p 1} & 0
\end{array}\right] \\
& \overline{\boldsymbol{B}}_{1}=\left[\begin{array}{ll}
\mathbf{o} & 1
\end{array}\right]^{T}, \overline{\boldsymbol{B}}_{2}=\left[\begin{array}{ll}
\boldsymbol{B}_{p}^{T} & 0
\end{array}\right]^{T} \\
& \overline{\boldsymbol{C}}_{10}=\left[\begin{array}{cc}
\boldsymbol{C}_{t 0} & 0 \\
\mathbf{0} & b_{s}
\end{array}\right], \overline{\boldsymbol{C}}_{11}=\left[\begin{array}{cc}
\boldsymbol{C}_{t 1} & 0 \\
\mathbf{0} & 0
\end{array}\right]
\end{aligned}
$$

なお, $\overline{\boldsymbol{E}}_{N}(\boldsymbol{x})$ は正則であるから, 制御量 $\overline{\boldsymbol{z}}$ は

$$
\begin{aligned}
& \bar{z}=\overline{\boldsymbol{C}}_{1 N}(\boldsymbol{x}) \boldsymbol{x}+\overline{\boldsymbol{D}}_{12 N}(\boldsymbol{x}) u \\
& \overline{\boldsymbol{C}}_{1 N}(\boldsymbol{x})=\overline{\boldsymbol{C}}_{10}+\overline{\boldsymbol{C}}_{11} \overline{\boldsymbol{E}}_{N}^{-1}(\boldsymbol{x}) \overline{\boldsymbol{A}}_{N}(\boldsymbol{x}) \\
& \overline{\boldsymbol{D}}_{12 N}(\boldsymbol{x})=\overline{\boldsymbol{C}}_{11} \overline{\boldsymbol{E}}_{N}^{-1}(\boldsymbol{x}) \overline{\boldsymbol{B}}_{2}
\end{aligned}
$$

のように表現することもできる.

\section{3 非線形 $\mathcal{H}_{\infty}$ 制御問題と固有值指定問題}

本論文ではディスクリプタ形式の非線形システム $\overline{\mathcal{S}}$ に対 して，以下に定義する非線形 $\mathcal{H}_{\infty}$ 制御問題を考える.

【定義】正定数 $\gamma$ が与えられたとき， $\mathcal{S}$ を安定化し，かつ

$$
\|\bar{z}\|_{2} \leq \gamma\|\bar{w}\|_{2}\left(\forall \bar{w} \in \mathcal{L}_{2} \cap \mathcal{L}_{\infty}^{c}\right)
$$

を満足する非線形状態フィードバック

$$
\mathcal{K}: u=\boldsymbol{K}(\boldsymbol{x}) \boldsymbol{x}
$$

が存在するならば，それを求める問題を非線形 $\mathcal{H}_{\infty}$ 制御問 題という.

非線形 $\mathcal{H}_{\infty}$ 制御問題の可解条件は， SLMI に基づくもの が知られているが ${ }^{18)}, \overline{\boldsymbol{E}}_{N}(\boldsymbol{x}), \overline{\boldsymbol{A}}_{N}(\boldsymbol{x})$ が $\boldsymbol{x}$ に関して有限 のべき級数ではないため, 直接この可解条件を用いることは できない.そこで本論文では, $\overline{\boldsymbol{E}}_{N}(\boldsymbol{x}), \overline{\boldsymbol{A}}_{N}(\boldsymbol{x})$ を $\boldsymbol{x}=\mathrm{o}$ で Taylor 級数展開することによって，

$$
\begin{aligned}
& \overline{\boldsymbol{E}}_{N}(\boldsymbol{x})=\overline{\boldsymbol{E}}(\boldsymbol{x})+\Delta \overline{\boldsymbol{E}}(\boldsymbol{x}), \overline{\boldsymbol{A}}_{N}(\boldsymbol{x})=\overline{\boldsymbol{A}}(\boldsymbol{x})+\Delta \overline{\boldsymbol{A}}(\boldsymbol{x}) \\
& \overline{\boldsymbol{E}}(\boldsymbol{x})=\overline{\boldsymbol{E}}_{(0)}+x_{3}^{2} \overline{\boldsymbol{E}}_{(33)} \\
& \overline{\boldsymbol{A}}(\boldsymbol{x})=\overline{\boldsymbol{A}}_{(0)}+x_{3}^{2} \overline{\boldsymbol{A}}_{(33)}+x_{4}^{2} \overline{\boldsymbol{A}}_{(44)}
\end{aligned}
$$$$
\Delta \overline{\boldsymbol{E}}(\boldsymbol{x})=\left[\begin{array}{ccccc}
0 & 0 & 0 & 0 & 0 \\
0 & 0 & 0 & \delta_{1}\left(x_{3}\right) & 0 \\
0 & 0 & 0 & 0 & 0 \\
0 & \delta_{2}\left(x_{3}\right) & 0 & 0 & 0 \\
0 & 0 & 0 & 0 & 0
\end{array}\right]
$$$$
\Delta \overline{\boldsymbol{A}}(\boldsymbol{x})=\left[\begin{array}{ccccc}
0 & 0 & 0 & 0 & 0 \\
0 & 0 & \delta_{3}\left(x_{3}, x_{4}\right) & 0 & 0 \\
0 & 0 & 0 & 0 & 0 \\
0 & 0 & \delta_{4}\left(x_{3}\right) & 0 & 0 \\
0 & 0 & 0 & 0 & 0
\end{array}\right]
$$$$
\delta_{1}\left(x_{3}\right)=\delta_{2}\left(x_{3}\right)=m \ell\left(\cos x_{3}-1+\frac{1}{2} x_{3}^{2}\right)
$$$$
\delta_{3}\left(x_{3}, x_{4}\right)=m \ell x_{4}^{2}\left(\frac{\sin x_{3}}{x_{3}}-1\right)
$$$$
\delta_{4}\left(x_{3}\right)=m \ell g\left(\frac{\sin x_{3}}{x_{3}}-1+\frac{1}{6} x_{3}^{2}\right)
$$

のように $\boldsymbol{x}$ に関して 2 次までの項 $\bar{E}(\boldsymbol{x}), \overline{\boldsymbol{A}}(\boldsymbol{x})$ と高次の項 $\Delta \bar{E}(x), \Delta \bar{A}(x)$ に分割し, 以下に定義する非線形ロバスト
$\mathcal{H}_{\infty}$ 制御問題を考えることにより (7) 式で表される $\overline{\mathcal{S}}$ に対 する非線形 $\mathcal{H}_{\infty}$ 制御問題を満足させることに注目する ${ }^{12)}$.

【定義】 $\boldsymbol{x}=\mathbf{o}$ を含む領域 $\mathcal{U}=\left\{\boldsymbol{x}|| x_{i} \mid \leq \bar{x}_{i}, i=\right.$ $3,4\}$ および正数 $\gamma$ が与えられたとき，Uにおいて構造的 不確かさ $\Delta \overline{\boldsymbol{E}}(\boldsymbol{x}), \Delta \overline{\boldsymbol{A}}(\boldsymbol{x})$ と関係せずに

$$
\overline{\mathcal{S}}:\left\{\begin{array}{l}
\begin{array}{l}
(\overline{\boldsymbol{E}}(\boldsymbol{x})+\Delta \overline{\boldsymbol{E}}(\boldsymbol{x})) \dot{\boldsymbol{x}}=(\overline{\boldsymbol{A}}(\boldsymbol{x})+\Delta \overline{\boldsymbol{A}}(\boldsymbol{x})) \boldsymbol{x} \\
\overline{\boldsymbol{z}}=\overline{\boldsymbol{C}}_{10} \boldsymbol{x}+\overline{\boldsymbol{C}}_{11} \dot{\boldsymbol{x}} \\
\boldsymbol{y}=\boldsymbol{x}
\end{array} \quad \overline{\boldsymbol{B}}_{1} \bar{w}+\overline{\boldsymbol{B}}_{2} u \\
\end{array}\right.
$$

を安定化し，かつ $\mathcal{L}_{2}$ ゲイン条件 (9) 式を満足する $\mathcal{K}$ が存 在するならばそれを求める問題を非線形ロバスト $\mathcal{H}_{\infty}$ 制御 問題という。

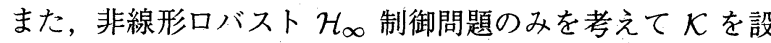
計するとゲインが過大となり，実用上好ましくないため，以 下に定義する固有值指定問題も考慮する。

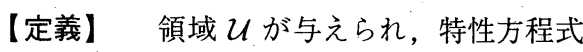

$$
\operatorname{det}\left[\lambda \overline{\boldsymbol{E}}(\boldsymbol{x})-\left(\overline{\boldsymbol{A}}(\boldsymbol{x})+\overline{\boldsymbol{B}}_{2} \boldsymbol{K}(\boldsymbol{x})\right)\right]=0
$$

の根を $\lambda=\alpha+j \beta$ としたとき， $(\alpha, \beta)$ を指定した範囲 (た とえば中心 $(q, 0)$, 半径 $r$ の円内) に配置する $\mathcal{K}$ が存在す るならばそれを求める問題を固有值指定問題という.

非線形システムの場合，極という概念が存在しないが，固 有値指定問題を考えることによって，線形システムにおける 極配置と同様の効果が期待できる.

\section{3. ディスクリプタ表現の柔軟性を利用しない場} 合の可解条件とその問題点

\section{1 非線形ロバスト $\mathcal{H}_{\infty}$ 制御問題の可解条件}

ここでの議論では, 簡単のため $b_{t 2}=0\left(\bar{C}_{11}=\mathbf{o}\right)$ とする. まず，

$$
\begin{aligned}
& \Delta \overline{\boldsymbol{E}}(\boldsymbol{x}) \overline{\boldsymbol{E}}^{-1}(\boldsymbol{x})=\left[\begin{array}{ccccc}
0 & 0 & 0 & 0 & 0 \\
0 & \delta_{11}\left(x_{3}\right) & 0 & \delta_{12}\left(x_{3}\right) & 0 \\
0 & 0 & 0 & 0 & 0 \\
0 & \delta_{21}\left(x_{3}\right) & 0 & \delta_{22}\left(x_{3}\right) & 0 \\
0 & 0 & 0 & 0 & 0
\end{array}\right] \\
& \delta_{11}\left(x_{3}\right)=-\frac{e\left(x_{3}\right) \delta_{1}\left(x_{3}\right)}{d_{E}\left(x_{3}\right)}, \delta_{12}\left(x_{3}\right)=\frac{\bar{M} \delta_{1}\left(x_{3}\right)}{d_{E}\left(x_{3}\right)} \\
& \delta_{21}\left(x_{3}\right)=\frac{\bar{J} \delta_{2}\left(x_{3}\right)}{d_{E}\left(x_{3}\right)}, \delta_{22}\left(x_{3}\right)=-\frac{e\left(x_{3}\right) \delta_{2}\left(x_{3}\right)}{d_{E}\left(x_{3}\right)} \\
& e\left(x_{3}\right)=m \ell\left(1-\frac{1}{2} x_{3}^{2}\right), d_{E}\left(x_{3}\right)=\bar{M} \bar{J}-e^{2}\left(x_{3}\right)
\end{aligned}
$$

における $\delta_{i j}\left(x_{3}\right)(i, j=1,2)$ および $\delta_{3}\left(x_{3}, x_{4}\right), \delta_{4}\left(x_{3}\right)$ が実用的な $\mathcal{U}$ で $\left|\delta_{i j}\left(x_{3}\right)\right| \leq\left|\delta_{i j}\left(\bar{x}_{3}\right)\right|,\left|\delta_{3}\left(x_{3}, x_{4}\right)\right| \leq$ $\left|\delta_{3}\left(\bar{x}_{3}, \bar{x}_{4}\right)\right|,\left|\delta_{4}\left(x_{3}\right)\right| \leq\left|\delta_{4}\left(\bar{x}_{3}\right)\right|$ であることを考慮し，

$$
\begin{aligned}
& \left\{\begin{array}{l}
\Delta \overline{\boldsymbol{E}}(\boldsymbol{x}) \overline{\boldsymbol{E}}^{-1}(\boldsymbol{x})=\widehat{\boldsymbol{M}}_{E} \widehat{\boldsymbol{\Sigma}}_{E}(\boldsymbol{x}) \widehat{\boldsymbol{N}}_{E} \\
\Delta \overline{\boldsymbol{A}}(\boldsymbol{x})=\boldsymbol{M}_{A} \boldsymbol{\Sigma}_{A}(\boldsymbol{x}) \boldsymbol{N}_{A}
\end{array}\right. \\
& \widehat{\boldsymbol{\Sigma}}_{E}^{T}(\boldsymbol{x}) \widehat{\boldsymbol{\Sigma}}_{E}(\boldsymbol{x}) \leq \boldsymbol{I}_{4}, \boldsymbol{\Sigma}_{A}^{T}(\boldsymbol{x}) \boldsymbol{\Sigma}_{A}(\boldsymbol{x}) \leq \boldsymbol{I}_{2}(\boldsymbol{x} \in \mathcal{U})
\end{aligned}
$$




$$
\widehat{\boldsymbol{M}}_{E}=\left[\begin{array}{cccc}
0 & 0 & 0 & 0 \\
\frac{\delta_{11}\left(\bar{x}_{3}\right)}{n_{11}} & \frac{\delta_{12}\left(\bar{x}_{3}\right)}{n_{12}} & 0 & 0 \\
0 & 0 & 0 & 0 \\
0 & 0 & \frac{\delta_{21}\left(\bar{x}_{3}\right)}{n_{21}} & \frac{\delta_{22}\left(\bar{x}_{3}\right)}{n_{22}} \\
0 & 0 & 0 & 0
\end{array}\right]
$$$$
\widehat{\boldsymbol{\Sigma}}_{E}(\boldsymbol{x})=\operatorname{diag}\left\{\frac{\delta_{11}\left(x_{3}\right)}{\delta_{11}\left(\bar{x}_{3}\right)}, \frac{\delta_{12}\left(x_{3}\right)}{\delta_{12}\left(\bar{x}_{3}\right)}, \frac{\delta_{21}\left(x_{3}\right)}{\delta_{21}\left(\bar{x}_{3}\right)}, \frac{\delta_{22}\left(x_{3}\right)}{\delta_{22}\left(\bar{x}_{3}\right)}\right\}
$$$$
\widehat{\boldsymbol{N}}_{E}=\left[\begin{array}{ccccc}
0 & n_{11} & 0 & 0 & 0 \\
0 & 0 & 0 & n_{12} & 0 \\
0 & n_{21} & 0 & 0 & 0 \\
0 & 0 & 0 & n_{22} & 0
\end{array}\right]
$$$$
\boldsymbol{M}_{A}=\left[\begin{array}{ccccc}
0 & \frac{\delta_{3}\left(\bar{x}_{3}, \bar{x}_{4}\right)}{n_{3}} & 0 & 0 & 0 \\
0 & 0 & 0 & \frac{\delta_{4}\left(\bar{x}_{3}\right)}{n_{4}} & 0
\end{array}\right]^{T}
$$$$
\boldsymbol{\Sigma}_{A}(\boldsymbol{x})=\operatorname{diag}\left\{\frac{\delta_{3}\left(x_{3}, x_{4}\right)}{\delta_{3}\left(\bar{x}_{3}, \bar{x}_{4}\right)}, \frac{\delta_{4}\left(x_{3}\right)}{\delta_{4}\left(\bar{x}_{3}\right)}\right\}
$$$$
N_{A}=\left[\begin{array}{lllll}
0 & 0 & n_{3} & 0 & 0 \\
0 & 0 & n_{4} & 0 & 0
\end{array}\right]
$$

のようにノルム有界型で表す ${ }^{12)}\left(n_{i j}, n_{k}\right.$ は正数).なお， $\Delta \overline{\boldsymbol{E}}(\boldsymbol{x})$ ではなく $\Delta \overline{\boldsymbol{E}}(\boldsymbol{x}) \overline{\boldsymbol{E}}^{-1}(\boldsymbol{x})$ をノルム有界型で表したの は，後述の SLMI を $\boldsymbol{x}$ に関する有限のべき級数とするため である。このとき，評価出力 $\boldsymbol{z}$ ，外生信号 $\boldsymbol{w}$ をそれぞれ

$$
\begin{aligned}
& \boldsymbol{z}=\boldsymbol{V}_{z}^{-1 / 2}\left[\begin{array}{c}
\overline{\boldsymbol{z}} \\
\tilde{\boldsymbol{z}}
\end{array}\right], \boldsymbol{w}=\boldsymbol{V}_{w}^{-1 / 2}\left[\begin{array}{c}
\bar{w} \\
\widetilde{\boldsymbol{w}}
\end{array}\right] \\
& \widetilde{\boldsymbol{z}}=\gamma\left[\begin{array}{c}
\widehat{\boldsymbol{N}}_{E} \overline{\boldsymbol{E}}(\boldsymbol{x}) \dot{\boldsymbol{x}} \\
\boldsymbol{N}_{A} \boldsymbol{x}
\end{array}\right], \widetilde{\boldsymbol{w}}=\left[\begin{array}{c}
\widehat{\boldsymbol{\Sigma}}_{E}(\boldsymbol{x}) \widehat{\boldsymbol{N}}_{E} \overline{\boldsymbol{E}}(\boldsymbol{x}) \dot{\boldsymbol{x}} \\
\boldsymbol{\Sigma}_{A}(\boldsymbol{x}) \boldsymbol{N}_{A} \boldsymbol{x}
\end{array}\right] \\
& \boldsymbol{V}_{z}=\operatorname{blockdiag}\left\{\boldsymbol{I}_{2}, \widetilde{\boldsymbol{V}}_{E}, \widetilde{\boldsymbol{V}}_{A}\right\} \\
& \boldsymbol{V}_{w}=\operatorname{blockdiag}\left\{1, \widetilde{\boldsymbol{V}}_{E}, \widetilde{\boldsymbol{V}}_{A}\right\} \\
& \widetilde{\boldsymbol{V}}_{E}=\operatorname{diag}\left\{\widetilde{v}_{11}, \widetilde{v}_{12}, \widetilde{v}_{21}, \widetilde{v}_{22}\right\}>0 \\
& \widetilde{\boldsymbol{V}}_{A}=\operatorname{diag}\left\{\widetilde{v}_{3}, \widetilde{v}_{4}\right\}>0
\end{aligned}
$$

とした非線形システム

$$
\begin{aligned}
& \mathcal{S}:\left\{\begin{array}{c}
\boldsymbol{E}(\boldsymbol{x}) \dot{\boldsymbol{x}}=\boldsymbol{A}(\boldsymbol{x}) \boldsymbol{x}+\boldsymbol{B}_{1} \boldsymbol{V}_{w}^{1 / 2} \boldsymbol{w}+\boldsymbol{B}_{2} u \\
\boldsymbol{V}_{z}^{1 / 2} \boldsymbol{z}=\boldsymbol{C}_{1}(\boldsymbol{x}) \boldsymbol{x}+\boldsymbol{D}_{11} \boldsymbol{V}_{w}^{1 / 2} \boldsymbol{w}+\boldsymbol{D}_{12} u
\end{array}\right. \\
& \boldsymbol{E}(\boldsymbol{x})=\overline{\boldsymbol{E}}(\boldsymbol{x}), \boldsymbol{A}(\boldsymbol{x})=\overline{\boldsymbol{A}}(\boldsymbol{x}) \\
& \boldsymbol{B}_{1}=\left[\begin{array}{lll}
\overline{\boldsymbol{B}}_{1} & -\widehat{\boldsymbol{M}}_{E} & \boldsymbol{M}_{A}
\end{array}\right], \boldsymbol{B}_{2}=\overline{\boldsymbol{B}}_{2} \\
& \boldsymbol{C}_{1}(\boldsymbol{x})=\left[\begin{array}{lll}
\overline{\boldsymbol{C}}_{10}^{T} & \left(\gamma \widehat{\boldsymbol{N}}_{E} \overline{\boldsymbol{A}}(\boldsymbol{x})\right)^{T} & \left(\gamma \boldsymbol{N}_{A}\right)^{T}
\end{array}\right]^{T} \\
& \boldsymbol{D}_{11}=\left[\begin{array}{ccc}
\boldsymbol{O}_{2 \times 1} & \boldsymbol{O}_{2 \times 4} & \boldsymbol{O}_{2 \times 2} \\
\widehat{\boldsymbol{N}}_{E} \overline{\boldsymbol{B}}_{1} & -\gamma \widehat{\boldsymbol{N}}_{E} \widehat{\boldsymbol{M}}_{E} & \gamma \widehat{\boldsymbol{N}}_{E} \boldsymbol{M}_{A} \\
\boldsymbol{O}_{2 \times 1} & \boldsymbol{O}_{2 \times 4} & \boldsymbol{O}_{2 \times 2}
\end{array}\right] \\
& \boldsymbol{D}_{12}=\left[\begin{array}{lll}
\boldsymbol{O}_{2 \times 1}^{T} & \left(\gamma \widehat{\boldsymbol{N}}_{E} \overline{\boldsymbol{B}}_{2}\right)^{T} & \boldsymbol{O}_{2 \times 1}^{T}
\end{array}\right]^{T}
\end{aligned}
$$

に対する $\mathcal{L}_{2}$ ゲイン条件を考えることによって，(11) 式で表 される $\overline{\mathcal{S}}$ に対する非線形ロバスト $\mathcal{H}_{\infty}$ 制御問題の可解条件
が得られる．以下に文献 12) で示した結果を SLMI の解が $\boldsymbol{x}$ に依存した場合に拡張した定理を示す。

《定理 1》 正数 $\gamma$ が与えられ，領域 $U$ で sLMI

$$
\begin{gathered}
\boldsymbol{Q}_{\mathcal{L}_{2}}(\boldsymbol{x}):=\left[\begin{array}{cc}
-\operatorname{He}\left[\boldsymbol{\Theta}_{\mathcal{L}_{2}}(\boldsymbol{x})\right]+\boldsymbol{E}(\boldsymbol{x}) \dot{\boldsymbol{\Phi}}(\boldsymbol{x}) \boldsymbol{E}^{T}(\boldsymbol{x}) \\
\boldsymbol{V}_{w} \boldsymbol{B}_{1}^{T} \\
\left(\boldsymbol{C}_{1}(\boldsymbol{x}) \boldsymbol{\Phi}(\boldsymbol{x})+\boldsymbol{D}_{12} \boldsymbol{Z}(\boldsymbol{x})\right) \boldsymbol{E}^{T}(\boldsymbol{x}) \\
\star & \star \\
\gamma^{2} \boldsymbol{V}_{w} & \star \\
-\boldsymbol{D}_{11} \boldsymbol{V}_{w} & \boldsymbol{V}_{z}
\end{array}\right]>0 \\
\boldsymbol{\Theta}_{\mathcal{L}_{2}}(\boldsymbol{x})=\left(\boldsymbol{A}(\boldsymbol{x}) \boldsymbol{\Phi}(\boldsymbol{x})+\boldsymbol{B}_{2} \boldsymbol{Z}(\boldsymbol{x})\right) \boldsymbol{E}^{T}(\boldsymbol{x})
\end{gathered}
$$

を満足する $\boldsymbol{\Phi}(\boldsymbol{x})=\boldsymbol{\Phi}^{T}(\boldsymbol{x})>0, \widetilde{\boldsymbol{V}}_{E}>0, \widetilde{\boldsymbol{V}}_{A}>0, \boldsymbol{Z}(\boldsymbol{x})$ が存在するとき, $\boldsymbol{K}(\boldsymbol{x})=\boldsymbol{Z}(\boldsymbol{x}) \boldsymbol{\Phi}(\boldsymbol{x})^{-1}$ は (11) 式で表され る $\overline{\mathcal{S}}$ に対する非線形ロバスト $\mathcal{H}_{\infty}$ 制御問題の解である.

(証明) 付録 $\mathbf{A}$ を参照のこと.

\section{2 円内への固有値指定問題の可解条件}

文献 2) で示されている不確かなパラメー夕に依存した線 形システムに対する極配置問題の可解条件を利用すると，円 内への固有値指定問題の可解条件は以下のようになる.

《定理 2》円の中心 $(q, 0)$, 半径 $r$ が与えられたとき, sLMI

$$
\begin{gathered}
\boldsymbol{Q}_{e i g}(\boldsymbol{x}):=\left[\begin{array}{c}
q \mathrm{He}\left[\boldsymbol{\Theta}_{e i g}(\boldsymbol{x})\right]+d \overline{\boldsymbol{E}}(\boldsymbol{x}) \boldsymbol{\Phi}(\boldsymbol{x}) \overline{\boldsymbol{E}}^{T}(\boldsymbol{x}) \\
\boldsymbol{\Theta}_{e i g}^{T}(\boldsymbol{x}) \\
\star \\
\overline{\boldsymbol{E}}(\boldsymbol{x}) \boldsymbol{\Phi}(\boldsymbol{x}) \overline{\boldsymbol{E}}^{T}(\boldsymbol{x})
\end{array}\right]>0 \\
\boldsymbol{\Theta}_{e i g}(\boldsymbol{x}):=\left(\overline{\boldsymbol{A}}(\boldsymbol{x}) \boldsymbol{\Phi}(\boldsymbol{x})+\overline{\boldsymbol{B}}_{2} \boldsymbol{Z}(\boldsymbol{x})\right) \overline{\boldsymbol{E}}^{T}(\boldsymbol{x})
\end{gathered}
$$

を満足する $\boldsymbol{\Phi}(\boldsymbol{x})=\boldsymbol{\Phi}^{T}(\boldsymbol{x})>0$ および $\boldsymbol{Z}(\boldsymbol{x})$ が領域 $\mathcal{U}$ で存 在するとき, $\boldsymbol{K}(\boldsymbol{x})=\boldsymbol{Z}(\boldsymbol{x}) \boldsymbol{\Phi}^{-1}(\boldsymbol{x})$ は非線形システム $\overline{\mathcal{S}}$ に 対する中心 $(q, 0)$, 半径 $r$ の円内への固有値指定問題を満足 する.ただし $d=r^{2}-q^{2}$ である.

\section{3 ディスクリプタ表現の柔軟性を利用しない場合の問}

\section{題点}

定理 1，2 で示した結果では，以下のような問題がある. 問題点 $1 \quad \bar{C}_{11} \neq \mathbf{o}\left(b_{t 2} \neq 0\right)$ のとき，(8) 式の評価出力 $\overline{\boldsymbol{z}}$ は $\overline{\boldsymbol{E}}_{N}^{-1}(\boldsymbol{x})$ を含むため, $\overline{\boldsymbol{C}}_{1 N}(\boldsymbol{x})=\overline{\boldsymbol{C}}_{1}(\boldsymbol{x})+\Delta \overline{\boldsymbol{C}}_{1}(\boldsymbol{x})$, $\overline{\boldsymbol{D}}_{12 N}(\boldsymbol{x})=\overline{\boldsymbol{D}}_{12}(\boldsymbol{x})+\Delta \overline{\boldsymbol{D}}_{12}(\boldsymbol{x})$ と表したときの $\Delta \overline{\boldsymbol{C}}_{1}(\boldsymbol{x})$, $\Delta \bar{D}_{12}(x)$ をノルム有界型で表すと見積もりが保守的になる.

問題点 $2 \quad \Delta \bar{E}(x)$ ではなく $\Delta \bar{E}(x) \bar{E}^{-1}(x)$ をノルム有界 型で表しているため, 線形ロバスト $\mathcal{H}_{\infty}$ 制御問題を考え た場合 ${ }^{1)}$ と比べて構造的不確かさに起因する外生信号 $\widetilde{\boldsymbol{w}}$, 評価出力 $\widetilde{z}$ の数が増える.

問題点 $3 \quad \Phi(x), Z(x)$ を

$$
\boldsymbol{\Phi}(\boldsymbol{x})=\boldsymbol{\Phi}_{(0)}, \boldsymbol{Z}(\boldsymbol{x})=\boldsymbol{Z}_{(0)}+x_{3}^{2} \boldsymbol{Z}_{(33)}
$$

という単純な形式としても $\overline{\boldsymbol{E}}(\boldsymbol{x})$ が $x_{3}^{2}$ に依存しているた め, sLMI (16), (17) 式は

$$
\begin{aligned}
& \text { blockdiag }\left\{\boldsymbol{\Phi}(\boldsymbol{x}), \widetilde{\boldsymbol{V}}_{A}, \widetilde{\boldsymbol{V}}_{\boldsymbol{E}}, \boldsymbol{Q}_{\mathcal{L}_{2}}(\boldsymbol{x}), \boldsymbol{Q}_{\text {eig }}(\boldsymbol{x})\right\} \\
= & \boldsymbol{Q}_{(0)}+x_{3}^{2} \boldsymbol{Q}_{(33)}+x_{4}^{2} \boldsymbol{Q}_{(44)}
\end{aligned}
$$




$$
+x_{3}^{4} \boldsymbol{Q}_{(3333)}+x_{3}^{2} x_{4}^{2} \boldsymbol{Q}_{(3344)}>0
$$

のように $x_{3}^{2}$ に関して 2 次, $x_{4}^{2}$ に関して 1 次となる. そのため, 変動 $\mathcal{T}_{3}=\left\{\left(x_{3}^{2}, x_{3}^{4}\right)|| x_{3} \mid \leq \bar{x}_{3}\right\}, \mathcal{T}_{4}=$ $\left\{x_{4}^{2}|| x_{4} \mid \leq \bar{x}_{4}\right\}$ を囲う凸多面体 $\mathcal{H}_{3}, \mathcal{H}_{4}$ の頂点で (19) 式における $\left(x_{3}^{2}, x_{3}^{4}, x_{4}^{2}\right)$ を置き換えた LMI を解けば よいが 10),12)， $\mathcal{T}_{3}$ は曲線であるため $\mathcal{T}_{3}$ を保守性なく $\mathcal{H}_{3}$ で囲うことができない.

このように,ディスクリプタ変数 (状態変数) の時間微分 $\dot{\boldsymbol{x}}$ の係数行列 $\bar{E}(\boldsymbol{x})$ が $x_{3}^{2}$ に依存しているため, 定理 1,2 に 示した結果を用いると保守的になる3).

\section{4. ディスクリプタ表現の柔軟性を利用した可解条件}

\section{1 $\dot{x}$ をディスクリプタ変数とした場合の $\overline{\mathcal{S}}$}

ここでは, 3.3 で述べた柔軟性を利用しない場合の問題 点に対処するため, ディスクリプタ表現の柔軟性を利用し， ディスクリプタ変数 $\boldsymbol{\xi}$ の時間微分 $\dot{\boldsymbol{\xi}}$ の係数行列 $\overline{\boldsymbol{E}}^{\sharp}$ が $\boldsymbol{x}$ に 依存しないような変換を行う ${ }^{4), 5), 15) . ~}$

\section{ディスクリプタ変数を}

$$
\xi=\left[\begin{array}{ll}
\boldsymbol{x}^{T} & \dot{x}^{T}
\end{array}\right]^{T}
$$

と定義し，(11)，(10) 式で表される $\overline{\mathcal{S}} ， \mathcal{K}$ を書き換えると，

$$
\begin{aligned}
& \overline{\mathcal{S}}:\left\{\begin{array}{l}
\overline{\boldsymbol{E}}^{\sharp} \dot{\boldsymbol{\xi}}=\left(\overline{\boldsymbol{A}}^{\sharp}(\boldsymbol{x})+\Delta \overline{\boldsymbol{A}}^{\sharp}(\boldsymbol{x})\right) \boldsymbol{\xi} \\
\overline{\boldsymbol{z}}=\overline{\boldsymbol{C}}_{1}^{\sharp} \boldsymbol{\xi} \\
\boldsymbol{y}=\boldsymbol{x}
\end{array}\right. \\
& \mathcal{K}: u=\boldsymbol{K}^{\sharp}(\boldsymbol{x}) \boldsymbol{\xi} \\
& \overline{\boldsymbol{E}}^{\sharp}=\left[\begin{array}{cc}
\boldsymbol{I}_{5} & \boldsymbol{O}_{5 \times 5} \\
\boldsymbol{O}_{5 \times 5} & \boldsymbol{O}_{5 \times 5}
\end{array}\right] \\
& \bar{A}^{\sharp}(\boldsymbol{x})=\left[\begin{array}{cc}
\boldsymbol{O}_{5 \times 5} & \boldsymbol{I}_{5} \\
\overline{\boldsymbol{A}}(\boldsymbol{x}) & -\overline{\boldsymbol{E}}(\boldsymbol{x})
\end{array}\right] \\
& \Delta \overline{\boldsymbol{A}}^{\sharp}(\boldsymbol{x})=\left[\begin{array}{cc}
\boldsymbol{O}_{5 \times 5} & \boldsymbol{O}_{5 \times 5} \\
\Delta \overline{\boldsymbol{A}}(\boldsymbol{x}) & -\Delta \overline{\boldsymbol{E}}(\boldsymbol{x})
\end{array}\right] \\
& \bar{B}_{1}^{\sharp}=\left[\begin{array}{c}
\boldsymbol{O}_{5 \times 1} \\
\bar{B}_{1}
\end{array}\right], \bar{B}_{2}^{\sharp}=\left[\begin{array}{c}
\boldsymbol{O}_{5 \times 1} \\
\bar{B}_{2}
\end{array}\right] \\
& \overline{\boldsymbol{C}}_{1}^{\sharp}=\left[\begin{array}{ll}
\overline{\boldsymbol{C}}_{10} & \overline{\boldsymbol{C}}_{11}
\end{array}\right], \boldsymbol{K}^{\sharp}(\boldsymbol{x})=\left[\begin{array}{ll}
\boldsymbol{K}(\boldsymbol{x}) & \boldsymbol{O}_{1 \times 5}
\end{array}\right]
\end{aligned}
$$

となる.このとき，ディスクリプタ変数の時間微分 $\dot{\boldsymbol{\xi}}$ の係数 行列 $\overline{\boldsymbol{E}}^{\sharp}$ は定数行列となる.さらに, 制御量 $\overline{\boldsymbol{z}}$ を (8) 式の ように $\boldsymbol{x}$ と $u$ を用いて表す代わりに，(7) 式のように $\boldsymbol{x}$ と $\dot{\boldsymbol{x}}$ を用いて表すことによって，構造的不確かさを陽に含まな い $\overline{\boldsymbol{z}}=\overline{\boldsymbol{C}}_{1}^{\sharp} \boldsymbol{\xi}$ という簡単な形式となり，柔軟性を利用しない 場合の問題点 1 に対処できる.

また，実用的な $\mathcal{U}$ で $\left|\delta_{i}\left(x_{3}\right)\right| \leq\left|\delta_{i}\left(\bar{x}_{3}\right)\right|(i=1,2,4)$ ， $\left|\delta_{3}\left(x_{3}, x_{4}\right)\right| \leq\left|\delta_{3}\left(\bar{x}_{3}, \bar{x}_{4}\right)\right|$ となることを考慮し，柔軟性を 利用しない場合の問題点 2 に対処するために $\Delta \overline{\boldsymbol{E}}(\boldsymbol{x})$ を

$$
\begin{aligned}
& \Delta \overline{\boldsymbol{E}}(\boldsymbol{x})=\boldsymbol{M}_{E} \boldsymbol{\Sigma}_{E}(\boldsymbol{x}) \boldsymbol{N}_{E} \\
& \boldsymbol{\Sigma}_{E}^{T}(\boldsymbol{x}) \boldsymbol{\Sigma}_{E}(\boldsymbol{x}) \leq \boldsymbol{I}_{2}, \boldsymbol{\Sigma}_{A}^{T}(\boldsymbol{x}) \boldsymbol{\Sigma}_{A}(\boldsymbol{x}) \leq \boldsymbol{I}_{2}(\boldsymbol{x} \in \mathcal{U})
\end{aligned}
$$

$$
\begin{aligned}
& \boldsymbol{M}_{E}=\left[\begin{array}{ccccc}
0 & \frac{\delta_{1}\left(\bar{x}_{3}\right)}{n_{1}} & 0 & 0 & 0 \\
0 & 0 & 0 & \frac{\delta_{2}\left(\bar{x}_{3}\right)}{n_{2}} & 0
\end{array}\right]^{T} \\
& \boldsymbol{\Sigma}_{E}(\boldsymbol{x})=\operatorname{diag}\left\{\frac{\delta_{1}\left(x_{3}\right)}{\delta_{1}\left(\bar{x}_{3}\right)}, \frac{\delta_{2}\left(x_{3}\right)}{\delta_{2}\left(\bar{x}_{3}\right)}\right\} \\
& \boldsymbol{N}_{E}=\left[\begin{array}{ccccc}
0 & 0 & 0 & n_{1} & 0 \\
0 & n_{2} & 0 & 0 & 0
\end{array}\right]
\end{aligned}
$$

と表し，また， $\Delta \overline{\boldsymbol{A}}(\boldsymbol{x})$ を(13) 式のようにノルム有界型で表 すと, 構造的不確かさ $\Delta \overline{\boldsymbol{A}}^{\sharp}(\boldsymbol{x})$ は

$$
\begin{aligned}
\Delta \bar{A}^{\sharp}(x) & =M_{A}^{\sharp} \boldsymbol{\Sigma}_{A}^{\sharp}(x) N_{A}^{\sharp}, \boldsymbol{\Sigma}_{A}^{\sharp T}(\boldsymbol{x}) \boldsymbol{\Sigma}_{A}^{\sharp}(\boldsymbol{x}) \leq \boldsymbol{I}_{4} \\
\boldsymbol{M}_{A}^{\sharp} & =\left[\begin{array}{cc}
\boldsymbol{O}_{5 \times 2} & \boldsymbol{O}_{5 \times 2} \\
M_{A} & -\boldsymbol{M}_{E}
\end{array}\right] \\
\boldsymbol{\Sigma}_{A}^{\sharp}(\boldsymbol{x}) & =\operatorname{blockdiag}\left\{\boldsymbol{\Sigma}_{A}(\boldsymbol{x}), \boldsymbol{\Sigma}_{E}(\boldsymbol{x})\right\} \\
\boldsymbol{N}_{A}^{\sharp} & =\left[\begin{array}{cc}
\boldsymbol{N}_{A} & \boldsymbol{O}_{2 \times 5} \\
\boldsymbol{O}_{2 \times 5} & \boldsymbol{N}_{E}
\end{array}\right]
\end{aligned}
$$

と表される。ただし， $n_{i}(i=1, \cdots, 4)$ は適当な正数である.

\section{2 非線形ロバスト $\mathcal{H}_{\infty}$ 制御問題の可解条件}

構造的不確かさ $\Delta \overline{\boldsymbol{E}}(\boldsymbol{x}), \Delta \overline{\boldsymbol{A}}(\boldsymbol{x})$ に起因する外生信号を $\widetilde{\boldsymbol{w}}^{\sharp}=\boldsymbol{\Sigma}_{A}^{\sharp}(\boldsymbol{x}) \boldsymbol{N}_{A}^{\sharp} \boldsymbol{\xi}$ とおくと, (21) 式より

$$
\overline{\boldsymbol{E}}^{\sharp} \dot{\boldsymbol{\xi}}=\overline{\boldsymbol{A}}^{\sharp}(\boldsymbol{x}) \boldsymbol{\xi}+\left[\begin{array}{cc}
\overline{\boldsymbol{B}}_{1}^{\sharp} & \boldsymbol{M}_{\boldsymbol{A}}^{\sharp}
\end{array}\right]\left[\begin{array}{c}
\bar{w} \\
\tilde{\boldsymbol{w}}^{\sharp}
\end{array}\right]+\overline{\boldsymbol{B}}_{2}^{\sharp} u(24)
$$

となる。したがって, 領域 Uにおいて $\widetilde{\boldsymbol{w}}^{\sharp}$ から評価出力 $\widetilde{z}^{\sharp}=\gamma \boldsymbol{N}_{A}^{\sharp} \boldsymbol{\xi}$ までの $\mathcal{L}_{2}$ ゲインを $\gamma$ 以下にするような $\mathcal{K}$ は 構造的不確かさ $\Delta \overline{\boldsymbol{E}}(\boldsymbol{x}), \Delta \overline{\boldsymbol{A}}(\boldsymbol{x})$ と無関係に $\overline{\mathcal{S}}$ を安定化す ると考えられる。 そこで, 評価出力 $\boldsymbol{z}^{\sharp}$, 外生信号 $\boldsymbol{w}^{\sharp}$

$$
\begin{aligned}
\boldsymbol{z}^{\sharp} & =\boldsymbol{S}_{z}^{-1 / 2}\left[\begin{array}{c}
\overline{\boldsymbol{z}} \\
\widetilde{\boldsymbol{z}}^{\sharp}
\end{array}\right], \boldsymbol{w}^{\sharp}=\boldsymbol{S}_{w}^{-1 / 2}\left[\begin{array}{c}
\bar{w} \\
\widetilde{\boldsymbol{w}}^{\sharp}
\end{array}\right] \\
\boldsymbol{S}_{z} & =\operatorname{blockdiag}\left\{\boldsymbol{I}_{2}, \widetilde{\boldsymbol{S}}_{A}, \widetilde{\boldsymbol{S}}_{E}\right\} \\
\boldsymbol{S}_{w} & =\operatorname{blockdiag}\left\{1, \widetilde{\boldsymbol{S}}_{A}, \widetilde{\boldsymbol{S}}_{E}\right\} \\
\widetilde{\boldsymbol{S}}_{E} & =\operatorname{diag}\left\{\widetilde{s}_{1}, \widetilde{s}_{2}\right\}>0, \widetilde{\boldsymbol{S}}_{A}=\operatorname{diag}\left\{\widetilde{s}_{3}, \widetilde{s}_{4}\right\}>0
\end{aligned}
$$

と定義すると，以下の非線形システムが得られる．

$$
\begin{aligned}
\mathcal{S}^{\sharp}:\left\{\begin{array}{l}
\boldsymbol{E}^{\sharp} \dot{\boldsymbol{\xi}}=\boldsymbol{A}^{\sharp}(\boldsymbol{x}) \boldsymbol{\xi}+\boldsymbol{B}_{1}^{\sharp} \boldsymbol{S}_{w}^{1 / 2} \boldsymbol{w}^{\sharp}+\boldsymbol{B}_{2}^{\sharp} u \\
\boldsymbol{S}_{z}^{1 / 2} \boldsymbol{z}^{\sharp}=C_{1}^{\sharp} \boldsymbol{\xi} \\
\boldsymbol{y}=\boldsymbol{x}
\end{array}\right. \\
\boldsymbol{E}^{\sharp}=\overline{\boldsymbol{E}}^{\sharp}, \boldsymbol{A}^{\sharp}(\boldsymbol{x})=\overline{\boldsymbol{A}}^{\sharp}(\boldsymbol{x}) \\
\boldsymbol{B}_{1}^{\sharp}=\left[\begin{array}{ll}
\overline{\boldsymbol{B}}_{1}^{\sharp} & \boldsymbol{M}_{\boldsymbol{A}}^{\sharp}
\end{array}\right], \boldsymbol{B}_{2}^{\sharp}=\overline{\boldsymbol{B}}_{2}^{\sharp}, \boldsymbol{C}_{1}^{\sharp}=\left[\begin{array}{c}
\overline{\boldsymbol{C}}_{1}^{\sharp} \\
\gamma \boldsymbol{N}_{A}^{\sharp}
\end{array}\right]
\end{aligned}
$$

ただし， $\widetilde{\boldsymbol{S}}_{A}, \widetilde{\boldsymbol{S}}_{E}$ はスケーリング行列である.

文献 5)，8，9）の結果を用いると，以下の定理で示すよ うに, 等式制約を含む SLMI が非線形ロバスト $\mathcal{H}_{\infty}$ 制御問 題の可解条件として得られる.

《定理 3》 $\mathcal{S}^{\sharp}$ および正数 $\gamma$ が与えられ, 領域 $U$ で sLMI

$$
\boldsymbol{E}^{\sharp} \boldsymbol{X}_{\mathcal{L}_{2}}^{T}(\boldsymbol{x})=\boldsymbol{X}_{\mathcal{L}_{2}}(\boldsymbol{x}) \boldsymbol{E}^{\sharp T} \geq 0
$$




$$
\begin{gathered}
\boldsymbol{Q}_{\mathcal{L}_{2}}^{\sharp}(\boldsymbol{x}):=\left[\begin{array}{cc}
-\mathrm{He}\left[\boldsymbol{\Theta}_{\mathcal{L}_{2}}^{\sharp}(\boldsymbol{x})\right]+\boldsymbol{E}^{\sharp} \dot{\boldsymbol{X}}_{\mathcal{L}_{2}}^{T}(\boldsymbol{x}) \\
\boldsymbol{S}_{w} \boldsymbol{B}_{1}^{\sharp T} \\
\boldsymbol{C}_{1}^{\sharp} \boldsymbol{X}_{\mathcal{L}_{2}}^{T}(\boldsymbol{x}) \\
\star & \star \\
\gamma^{2} \boldsymbol{S}_{w} & \star \\
\boldsymbol{O}_{6 \times 5} & \boldsymbol{S}_{z}
\end{array}\right]>0 \\
\boldsymbol{\Theta}_{\mathcal{L}_{2}}^{\sharp}(\boldsymbol{x})=\boldsymbol{A}^{\sharp}(\boldsymbol{x}) \boldsymbol{X}_{\mathcal{L}_{2}}^{T}(\boldsymbol{x})+\boldsymbol{B}_{2}^{\sharp} \boldsymbol{Z}^{\sharp}(\boldsymbol{x})
\end{gathered}
$$

を満足する

$$
\begin{aligned}
\boldsymbol{X}_{\mathcal{L}_{2}}(\boldsymbol{x}) & :=\left[\begin{array}{cc}
\boldsymbol{X}_{1}(\boldsymbol{x}) & \boldsymbol{X}_{\mathcal{L}_{2} 2}(\boldsymbol{x}) \\
\boldsymbol{O}_{5 \times 5} & \boldsymbol{X}_{\mathcal{L}_{2} 4}(\boldsymbol{x})
\end{array}\right] \\
\boldsymbol{Z}^{\sharp}(\boldsymbol{x}) & :=\left[\begin{array}{ll}
\boldsymbol{Z}_{1}(\boldsymbol{x}) & \boldsymbol{Z}_{2}(\boldsymbol{x})
\end{array}\right]
\end{aligned}
$$

および $\widetilde{\boldsymbol{S}}_{A}>0, \widetilde{\boldsymbol{S}}_{E}>0$ が存在するとする.ただし， $\boldsymbol{X}_{1}(\boldsymbol{x})$, $\boldsymbol{X}_{\mathcal{L}_{2} 2}(\boldsymbol{x}), \boldsymbol{X}_{\mathcal{L}_{2} 4}(\boldsymbol{x}) \in \mathcal{R}^{5 \times 5}, \boldsymbol{Z}_{1}(\boldsymbol{x}), \boldsymbol{Z}_{2}(\boldsymbol{x}) \in \mathcal{R}^{5}$ であり, $\boldsymbol{X}_{1}(\boldsymbol{x})=\boldsymbol{X}_{1}^{T}(\boldsymbol{x})>0, \boldsymbol{X}_{\mathcal{L}_{24}(\boldsymbol{x})}$ : 正則行列である。このと き, 非線形状態フィードバック

$$
\mathcal{K}: u=\boldsymbol{K}^{\sharp}(\boldsymbol{x}) \boldsymbol{\xi}, \boldsymbol{K}^{\sharp}(\boldsymbol{x}):=\boldsymbol{Z}^{\sharp}(\boldsymbol{x}) \boldsymbol{X}_{\mathcal{L}_{2}}^{-T}(\boldsymbol{x})
$$

は $\overline{\mathcal{S}}$ に対する非線形ロバスト $\mathcal{H}_{\infty}$ 制御問題の解である。ま た, sLMI (27), (28) 式を満足する $Z^{\sharp}(\boldsymbol{x})$ の構造を特に

$$
Z^{\sharp}(x):=\left[\begin{array}{ll}
Z_{1}(x) & O_{1 \times 5}
\end{array}\right]
$$

と限定すれば，非線形状態フィードバックは

$$
\mathcal{K}: u=\boldsymbol{K}(\boldsymbol{x}) \boldsymbol{x}, \boldsymbol{K}(\boldsymbol{x}):=\boldsymbol{Z}_{1}(\boldsymbol{x}) \boldsymbol{X}_{1}^{-1}(\boldsymbol{x})
$$

のように $\boldsymbol{x}$ のみに依存し，(22) 式の構造となる.

(証明) 付録 B を参照のこと.

(注意 1) $\boldsymbol{X}_{\mathcal{L}_{2}}(\boldsymbol{x})$ が非正則の場合, (28) 式の成立を損なわ ない範囲で $\boldsymbol{X}_{\mathcal{L}_{2}}(\boldsymbol{x})$ を $\boldsymbol{X}_{\mathcal{L}_{2}}(\boldsymbol{x})+\varepsilon \boldsymbol{I}(\varepsilon$ : 微小な正数 $)$ の ように微小変動させることにより， $\boldsymbol{X}_{\mathcal{L}_{2}}(\boldsymbol{x})+\varepsilon \boldsymbol{I}$ を正則に することができる ${ }^{8), 9)}$.また, $\boldsymbol{X}_{\mathcal{L}_{2}}(\boldsymbol{x})$ を $\boldsymbol{X}_{\mathcal{L}_{2}}(\boldsymbol{x})+\varepsilon \boldsymbol{I}$ で置き換えても (27) 式が成立する，そのため定理 3 では， 一般性を失うことなく $\boldsymbol{X}_{\mathcal{L}_{2}}(\boldsymbol{x})$ が正則であるとした，

(注意 2)（27）式には等式制約が含まれているため，このま までは MATLAB の LMI Toolbox を利用して厳密な解 を求めることは困難であるが，付録 $\mathrm{B}$ に示すように， $E^{\sharp}$ の構造に注意すると, (27) 式をこれと等価な等式制約のな い $\boldsymbol{X}_{1}(\boldsymbol{x})=\boldsymbol{X}_{1}^{T}(\boldsymbol{x})>0$ で置き換えることができる.

(注意 3) 上里ら ${ }^{6)}$ は $\boldsymbol{X}_{\mathcal{L}_{2}}(\boldsymbol{x})=\boldsymbol{E}^{\sharp} \boldsymbol{P}^{T}(\boldsymbol{x})+\boldsymbol{U} \boldsymbol{S}^{T} \boldsymbol{V}^{T}$ $\left(\boldsymbol{P}(\boldsymbol{x})=\boldsymbol{P}^{T}(\boldsymbol{x})>0, \boldsymbol{S}:\right.$ 正則行列, $\boldsymbol{U}, \boldsymbol{V}: \mathrm{Null}^{\sharp T}$, Null $\boldsymbol{E}^{\sharp}$ の基底からなる行列) とすれば (27) 式の等式制 約を取り除くことができ， $Z^{\sharp}(\boldsymbol{x})=\boldsymbol{L}(\boldsymbol{x}) \boldsymbol{E}^{\sharp T}$ という形式

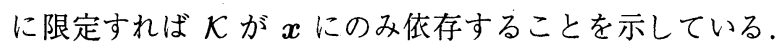
(26) 式の構造である場合, $\boldsymbol{X}_{\mathcal{L}_{2}}(\boldsymbol{x}), \boldsymbol{Z}^{\sharp}(\boldsymbol{x})$ の形式は本論 文で得られた結果と本質的に等価である。

\section{3 円内人の固有值指定問題の可解条件}

非線形 $\mathcal{H}_{\infty}$ 制御問題の可解条件を考慮すると, 円内への固有 值指定問題の場合も (17) 式における $\boldsymbol{\Theta}_{e i g}(\boldsymbol{x}), \overline{\boldsymbol{E}}(\boldsymbol{x}) \boldsymbol{\Phi}(\boldsymbol{x}) \overline{\boldsymbol{E}}^{T}$ をそれぞれ $\Theta_{e i g}^{\sharp}(x):=\bar{A}^{\sharp}(x) X_{e i g}^{T}(x)+\bar{B}_{2}^{\sharp} Z_{e i g}^{\sharp}(x)$,

$$
\overline{\boldsymbol{E}}^{\sharp} \boldsymbol{X}_{\text {eig }}^{T}(\boldsymbol{x})=\boldsymbol{X}_{\text {eig }}(x) \overline{\boldsymbol{E}}^{\sharp T} \geq 0
$$

で置き換えた sLMI

$$
\left[\begin{array}{cc}
q \mathrm{He}\left[\boldsymbol{\Theta}_{e i g}^{\sharp}(\boldsymbol{x})\right]+d \overline{\boldsymbol{E}}^{\sharp} \boldsymbol{X}_{e i g}^{T}(\boldsymbol{x}) & \star \\
\boldsymbol{\Theta}_{e i g}^{\sharp T}(\boldsymbol{x}) & \overline{\boldsymbol{E}}^{\sharp} \boldsymbol{X}_{e i g}^{T}(\boldsymbol{x})
\end{array}\right] \geq 0
$$

が可解条件であると考えられる。ただし， $d=r^{2}-q^{2}$ であ る。なお，(17) 式と異なり（35）式に等式制約がついている のは, (35) 式の $(2,2)$ ブロックである $\overline{\boldsymbol{E}}^{\sharp} \boldsymbol{X}_{e i g}^{T}(\boldsymbol{x})$ が半正定 行列であることを考虑したためである. 実際, 円内への固有 值指定問題の可解条件は以下の定理で与えられる.

《定理 4》 円の中心 $(q, 0)$, 半径 $r$ が与えられたとき, sLMI (34)，(35) 式を満足する

$$
\begin{aligned}
& \boldsymbol{X}_{e i g}(\boldsymbol{x}):=\left[\begin{array}{cc}
\boldsymbol{X}_{e i g 1}(\boldsymbol{x}) & \boldsymbol{X}_{\text {eig2 }}(\boldsymbol{x}) \\
\boldsymbol{O}_{5 \times 5} & \boldsymbol{X}_{\text {eig4 }}(\boldsymbol{x})
\end{array}\right] \\
& \boldsymbol{Z}_{\text {eig }}^{\sharp}(\boldsymbol{x}):=\left[\begin{array}{ll}
\boldsymbol{Z}_{\text {eig1 }}(\boldsymbol{x}) & \boldsymbol{Z}_{\text {eig2 }}(\boldsymbol{x})
\end{array}\right]
\end{aligned}
$$

が領域Uで存在するとする。ただし， $\boldsymbol{X}_{e i g 1}(\boldsymbol{x}), \boldsymbol{X}_{\text {eig2 }}(\boldsymbol{x})$, $\boldsymbol{X}_{\text {eig4 }}(\boldsymbol{x}) \in \mathcal{R}^{5 \times 5}, \boldsymbol{Z}_{\text {eig1 }}(\boldsymbol{x}), \boldsymbol{Z}_{\text {eig2 } 2}(\boldsymbol{x}) \in \mathcal{R}^{5}$ であり, $\boldsymbol{X}_{e i g 1}(\boldsymbol{x})=\boldsymbol{X}_{e i g 1}^{T}(\boldsymbol{x})>0, \boldsymbol{X}_{e i g 4}(\boldsymbol{x})$ : 正則行列である. このとき, 非線形状態フィードバック

$$
\mathcal{K}: u=\boldsymbol{K}^{\sharp}(\boldsymbol{x}) \boldsymbol{\xi}, \boldsymbol{K}^{\sharp}(\boldsymbol{x}):=\boldsymbol{Z}_{\text {eig }}^{\sharp}(\boldsymbol{x}) \boldsymbol{X}_{\text {eig }}^{-T}(\boldsymbol{x})
$$

は領域Uでディスクリプタ変数を $(20)$ 式としたシステム (21) 式に対する特性方程式

$$
\operatorname{det}\left[\lambda \overline{\boldsymbol{E}}^{\sharp}-\left(\overline{\boldsymbol{A}}^{\sharp}(\boldsymbol{x})+\overline{\boldsymbol{B}}_{2}^{\sharp} \boldsymbol{K}^{\sharp}(\boldsymbol{x})\right)\right]=0
$$

の根 $\lambda=\alpha+j \beta$ を中心 $(q, 0)$, 半径 $r$ の円内に配置する. また, sLMI (34), (35) 式を满足する $Z_{e i g}^{\sharp}(x)$ の構造を特に

$$
\boldsymbol{Z}_{\text {eig }}^{\sharp}(\boldsymbol{x}):=\left[\begin{array}{ll}
\boldsymbol{Z}_{\text {eig1 }}(\boldsymbol{x}) & \boldsymbol{O}_{1 \times 5}
\end{array}\right]
$$

と限定すれば，非線形状態フィードバックは

$$
\mathcal{K}: u=K(x) \boldsymbol{x}, \boldsymbol{K}(\boldsymbol{x}):=\boldsymbol{Z}_{\text {eig1 }}(\boldsymbol{x}) \boldsymbol{X}_{\text {eig1 }}^{-1}(\boldsymbol{x})
$$

のように $\boldsymbol{x}$ のみに依存した $(22)$ 式の構造となり，領域 U で元のシステム (11) 式に対する特性方程式 (12) 式の根 $\lambda=\alpha+j \beta$ を中心 $(q, 0)$, 半径 $r$ の円内に配置する.

(証明) 付録 $\mathbf{C}$ を参照のこと.

(注意 1) 定理 3 の注意 1 で述べた理由により，定理 4 で は一般性を失うことなく $\boldsymbol{X}_{e i g}(\boldsymbol{x})$ が正則であるとした

(注意 2) $\boldsymbol{Z}^{\sharp}(\boldsymbol{x}), \boldsymbol{Z}_{\text {eig }}^{\sharp}(\boldsymbol{x})$ の構造をそれぞれ (32), (40) 式 に限定すると, 非線形 $\mathcal{H}_{\infty}$ 制御問題と円内への固有值指定 問題を同時に満足する非線形状態フイードバックは (33), (41) 式より $\boldsymbol{Z}_{1}(\boldsymbol{x}) \boldsymbol{X}_{1}^{-1}(\boldsymbol{x})=\boldsymbol{Z}_{\text {eig1 }}(\boldsymbol{x}) \boldsymbol{X}_{\text {eig1 }}^{-1}(\boldsymbol{x})$ という制 約を満足する必要がある。したがって, $\boldsymbol{X}_{e i g 1}(\boldsymbol{x})=\boldsymbol{X}_{1}(\boldsymbol{x})$, $\boldsymbol{Z}_{\text {eig1 } 1}(\boldsymbol{x})=\boldsymbol{Z}_{1}(\boldsymbol{x})$ とすれば, 単に $\boldsymbol{X}_{\text {eig }}(\boldsymbol{x})=\boldsymbol{X}_{\mathcal{L}_{2}}(\boldsymbol{x})$, $Z_{\text {eig }}^{\sharp}(x)=Z_{\mathcal{L}_{2}}^{\sharp}(x)$ とするよりも解の保守性が軽減できる。

定理 3 の注意 2 で述べたうに，(34) 式はこれと等価な 等式制約のない $\boldsymbol{X}_{e i g 1}(\boldsymbol{x})=\boldsymbol{X}_{e i g 1}^{T}(\boldsymbol{x})>0$ で置き換えるこ とができるが, $\overline{\boldsymbol{E}}^{\sharp} \boldsymbol{X}_{\text {eig }}^{T}(\boldsymbol{x})$ が半正定行列であるため, 上里 らの結果 ${ }^{6}$ をを用いても (35) 式の等式制約を取り除くことは 
できない.そこで, 本論文では $\overline{\boldsymbol{E}}^{\sharp}$ の構造を考慮し, (35) 式 の等式制約を取り除いた以下の可解条件を提案する.

《定理 5》 円の中心 $(q, 0)$, 半径 $r$ が与えられたとき, sLMI

$$
\begin{aligned}
& \boldsymbol{Q}_{e i g}^{\sharp}(\boldsymbol{x}):=\left.\begin{array}{r}
q \mathrm{He}\left[\boldsymbol{\Theta}_{\text {eig }}^{\sharp}(\boldsymbol{x})\right]+d \overline{\boldsymbol{E}}^{\sharp} \boldsymbol{X}_{\text {eig }}^{T}(\boldsymbol{x}) \\
\boldsymbol{Q}_{\text {eig } 21}^{\sharp}(\boldsymbol{x}) \\
\star \\
\boldsymbol{X}_{1}(\boldsymbol{x})
\end{array}\right]>0 \quad(42) \\
& \boldsymbol{Q}_{\text {eig21 }}^{\sharp}(\boldsymbol{x}):=\left[\begin{array}{ll}
\boldsymbol{O}_{5 \times 5} & \left(\overline{\boldsymbol{A}}(\boldsymbol{x}) \boldsymbol{X}_{1}(\boldsymbol{x})+\overline{\boldsymbol{B}}_{2} \boldsymbol{Z}_{1}(\boldsymbol{x})\right)^{T}
\end{array}\right]
\end{aligned}
$$

を満足する

$$
\begin{aligned}
& \boldsymbol{X}_{e i g}(\boldsymbol{x}):=\left[\begin{array}{cc}
\boldsymbol{X}_{1}(\boldsymbol{x}) & \boldsymbol{X}_{\text {eig } 2}(\boldsymbol{x}) \\
\boldsymbol{O}_{5 \times 5} & \boldsymbol{X}_{\text {eig }}(\boldsymbol{x})
\end{array}\right] \\
& \boldsymbol{Z}_{\text {eig }}^{\sharp}(\boldsymbol{x}):=\left[\begin{array}{ll}
\boldsymbol{Z}_{1}(\boldsymbol{x}) & \boldsymbol{O}_{1 \times 5}
\end{array}\right]
\end{aligned}
$$

が領域 $U$ で存在するとする.ただし， $\boldsymbol{X}_{1}(\boldsymbol{x})=\boldsymbol{X}_{1}^{T}(\boldsymbol{x})>0$, $\boldsymbol{X}_{e i g 4}(\boldsymbol{x})$ : 正則行列, $d=r^{2}-q^{2}$ である.このとき, (33) 式 は領域 $\mathcal{U}$ で特性方程式 (12) 式の根 $\lambda=\alpha+j \beta$ を中心 $(q, 0)$, 半径 $r$ の円内に配置する.

(証明) 付録 D を参照のこと.

なお, 定理 5 は定理 4 の注意 2 を考慮した結果となってい ることに注意されたい。

\section{4 有限個の LMI への帰着}

4.2，4.3 の結果より sLMI (28) 式および (42) 式を同 時に満足する解 (29), (32), (43), (44) 式および $\widetilde{\boldsymbol{S}}_{A}>0$, $\widetilde{\boldsymbol{S}}_{E}>0$ を求めることができれば, 非線形 $\mathcal{H}_{\infty}$ 制御問題, 円 内への固有値指定問題を同時に満足し，かつ $\boldsymbol{x}$ にのみ依存 した非線形状態フィードバック (33) 式が得られる.

本論文では, sLMI (28), (42) 式を有限個の LMI に帰着 してその解を求めるため, 解 (29), (32), (43), (44) 式を

$$
\left\{\begin{array}{l}
\boldsymbol{X}_{1}(\boldsymbol{x})=\boldsymbol{X}_{1(0)} \\
\boldsymbol{X}_{\mathcal{L}_{2} 2}(\boldsymbol{x})=\boldsymbol{X}_{\mathcal{L}_{2} 2(0)}, \boldsymbol{X}_{\mathcal{L}_{2} 4}(\boldsymbol{x})=\boldsymbol{X}_{\mathcal{L}_{2} 4(0)} \\
\boldsymbol{X}_{e i g 2}(\boldsymbol{x})=\boldsymbol{X}_{e i g 2(0)}, \boldsymbol{X}_{e i g 4}(\boldsymbol{x})=\boldsymbol{X}_{e i g 4(0)} \\
\boldsymbol{Z}_{1}(\boldsymbol{x})=\boldsymbol{Z}_{1(0)}+x_{3}^{2} \boldsymbol{Z}_{1(33)}
\end{array}\right.
$$

という形式に選ぶ。なお, $\boldsymbol{Z}_{1}(\boldsymbol{x})=\boldsymbol{Z}_{1(0)}+x_{3}^{2} \boldsymbol{Z}_{1(33)}+$ $x_{4}^{2} Z_{1(44)}$ のように $Z_{1}(x)$ を $x_{4}^{2}$ に依存させることも考えら れるが，振子が台車に与える影響は十分小さいため，文献 12) で示したように $\boldsymbol{K}_{(44)}=\boldsymbol{Z}_{1(44)} \boldsymbol{X}_{1(0)}^{-1}$ は $\boldsymbol{K}_{(0)}, \boldsymbol{K}_{(33)}$ と比べて無視できるほど小さくなる。そこで本論文では $Z_{1(44)}=\boldsymbol{O}$ とした。 また， $\boldsymbol{X}_{1}(\boldsymbol{x})$ を $x_{3}^{2}$ に依存させ，

$$
\boldsymbol{X}_{1}(\boldsymbol{x})=\boldsymbol{X}_{1(0)}+x_{3}^{2} \boldsymbol{X}_{1(33)}
$$

とすることも考えられる．この場合， $\boldsymbol{X}_{1}(\boldsymbol{x})=\boldsymbol{X}_{1(0)}$ とし た場合と比べて $\bar{x}_{3}$ を大きく見積もることが可能となるが， 以下に示す理由で $\boldsymbol{X}_{1}(\boldsymbol{x})=\boldsymbol{X}_{1(0)}$ とした.

理由 $1 \boldsymbol{X}_{1}(\boldsymbol{x})$ を $(46)$ 式の形式とすると, $\dot{X}_{1}(\boldsymbol{x})=$ $2 x_{3} x_{4} \boldsymbol{X}_{1(33)}$ であるから sLMI は

$$
\begin{aligned}
& \operatorname{blockdiag}\left\{\boldsymbol{X}_{1}(\boldsymbol{x}), \widetilde{\boldsymbol{S}}_{A}, \widetilde{\boldsymbol{S}}_{E}, \boldsymbol{Q}_{\mathcal{L}_{2}}^{\sharp}(\boldsymbol{x}), \boldsymbol{Q}_{\text {eig }}^{\sharp}(\boldsymbol{x})\right\} \\
= & \boldsymbol{Q}_{(0)}^{\sharp}+x_{3}^{2} \boldsymbol{Q}_{(33)}^{\sharp}+x_{3} x_{4} \boldsymbol{Q}_{(34)}^{\sharp}
\end{aligned}
$$

$$
+x_{4}^{2} \boldsymbol{Q}_{(44)}^{\sharp}+x_{3}^{4} \boldsymbol{Q}_{(3333)}^{\sharp}+x_{3}^{2} x_{4}^{2} \boldsymbol{Q}_{(3344)}^{\sharp}>0(47)
$$

という形式となる。この場合, 保守性が少なくなるように $\mathcal{T}_{3}=\left\{\left(x_{3}, x_{3}^{2}, x_{3}^{4}\right)|| x_{3} \mid \leq \bar{x}_{3}\right\}$ を囲む凸多面体 $\mathcal{H}_{3}$ の頂 点を決めると, 頂点の数が増大し, 有限個の LMI の解を 求めるのに多大の時間を要する.

理由 $2 \boldsymbol{X}_{1}(\boldsymbol{x})$ を (46) 式の形式とすると, $\boldsymbol{K}(\boldsymbol{x})$ は複雑 な形式となり, 実用的ではない.

さらに, $\boldsymbol{X}_{\mathcal{L}_{2} 2}(\boldsymbol{x}), \boldsymbol{X}_{\mathcal{L}_{2} 4}(\boldsymbol{x}), \boldsymbol{X}_{e i g 2}(\boldsymbol{x}), \boldsymbol{X}_{\text {eig }}(\boldsymbol{x})$ を定数 行列としたのは, これらを $x_{3}^{2}$ に依存させると, (28), (42) 式 に打ける $\Theta_{\mathcal{L}_{2}}^{\sharp}(x), \Theta_{e i g}^{\sharp}(x)$ が $x_{3}^{2}$ に関して 2 次となり，柔 軟性を利用しない場合の問題点 3 に対処できないためである. sLMI (28)，(42) 式の解を（45）式の形式とすると, sLMI (28), (42) 式は $x_{3}^{2}, x_{4}^{2}$ に関して 1 次の sLMI

$$
\begin{aligned}
\boldsymbol{Q}^{\sharp}\left(x_{3}^{2}, x_{4}^{2}\right):=\operatorname{blockdiag}\left\{\boldsymbol{X}_{1}(\boldsymbol{x}), \widetilde{\boldsymbol{S}}_{A}, \widetilde{\boldsymbol{S}}_{E},\right. \\
\left.\boldsymbol{Q}_{\mathcal{L}_{2}}^{\sharp}(\boldsymbol{x}), \boldsymbol{Q}_{\text {eig }}^{\sharp}(\boldsymbol{x})\right\} \\
=\boldsymbol{Q}_{(0)}^{\sharp}+x_{3}^{2} \boldsymbol{Q}_{(33)}^{\sharp}+x_{4}^{2} \boldsymbol{Q}_{(44)}^{\sharp}>0
\end{aligned}
$$

となるため, 柔軟性を利用しない場合の問題点 3 に対処でき る.つまりこの場合, $\mathcal{T}_{i}=\left\{x_{i}^{2}|| x_{i} \mid \leq \bar{x}_{i}\right\}(i=3,4)$ を囲 む凸多面体 $\mathcal{H}_{i}$ の頂点は保守性なく $0, \bar{x}_{i}^{2}$ のように定まるた め, (48) 式の解を求める代わりに 4 個の LMI

$$
\left\{\begin{array}{l}
\boldsymbol{Q}^{\sharp}(0,0)=\boldsymbol{Q}_{(0)}^{\sharp}>0 \\
\boldsymbol{Q}^{\sharp}\left(\bar{x}_{3}^{2}, 0\right)=\boldsymbol{Q}_{(0)}^{\sharp}+\bar{x}_{3}^{2} \boldsymbol{Q}_{(33)}^{\sharp}>0 \\
\boldsymbol{Q}^{\sharp}\left(0, \bar{x}_{4}^{2}\right)=\boldsymbol{Q}_{(0)}^{\sharp}+\bar{x}_{4}^{2} \boldsymbol{Q}_{(44)}^{\sharp}>0 \\
\boldsymbol{Q}^{\sharp}\left(\bar{x}_{3}^{2}, \bar{x}_{4}^{2}\right)=\boldsymbol{Q}_{(0)}^{\sharp}+\bar{x}_{3}^{2} \boldsymbol{Q}_{(33)}^{\sharp}+\bar{x}_{4}^{2} \boldsymbol{Q}_{(44)}^{\sharp}>0
\end{array}\right.
$$

の共通解 (45) 式および $\widetilde{\boldsymbol{S}}_{A}, \widetilde{\boldsymbol{S}}_{E}$ を求めればよい ${ }^{15)}$ ．その 結果, 固有值指定条件付き非線形 $\mathcal{H}_{\infty}$ 状態フィードバック Kが以下のように求まる.

$$
\begin{aligned}
\mathcal{K}: u & =\left(\boldsymbol{K}_{(0)}+x_{3}^{2} \boldsymbol{K}_{(33)}\right) \boldsymbol{x} \\
\boldsymbol{K}_{(0)} & =\boldsymbol{Z}_{1(0)} \boldsymbol{X}_{1(0)}^{-1}, \boldsymbol{K}_{(33)}=\boldsymbol{Z}_{1(33)} \boldsymbol{X}_{1(0)}^{-1}
\end{aligned}
$$

\section{5. 設計例および実験結果}

\section{1 実験の条件}

本論文で提案した手法の有効性を検証するために, 実機実験 を行った. 非線形状態フィードバックは, $\gamma=1, b_{s}=1.1$ と して 3., 4. で提案した手法, 文献 16) で示した HamiltonJacobi 方程式 (不等式) に基づく手法により設計した。ただ L 3., 4. で提案した手法では, 固有值指定の設計パラメー 夕を $q=-12, r=11.5$ とし, $n_{i j}$ および $n_{k}$ を 0.001 , $\bar{x}_{4}=7.5 \bar{x}_{3}$ として LMI の解が求まる範囲で $\bar{x}_{3}$ をできるだ け大きくした．実験ではパラメー夕変動に対するロバスト性 を調べるため, 同定で用いた全長が $0.4[\mathrm{~m}]$ の振子の代わりに 全長が $0.6[\mathrm{~m}]$ のものを取り付け, 初期状態 $\boldsymbol{x}(0)=\mathbf{o}$ から振 子を倒立させたまま台車の位置 $r$ を台車の目標値 $r^{r e f}=1.8$ [m]に追従させた. 
$5.2 W_{t}(p)=b_{t 0}+b_{t 1} p\left(\overline{\boldsymbol{C}}_{11}=\mathbf{0}\right)$ の場合

重み関数を $W_{t}(p)=(p+0.5) / 5$ とした. 3. で提案した 手法では $\bar{x}_{3}=0.621$ 程度まで解が得られ(注 1 ), 固有值指定 条件付き非線形 $\mathcal{H}_{\infty}$ 状態フィードバック $\mathcal{K}_{\text {conv }}$ は以下のよ うになった。

$$
\begin{aligned}
\mathcal{K}_{\text {conv }}: u=\left(\boldsymbol{K}_{(0)}+x_{3}^{2} \boldsymbol{K}_{(33)}\right) \boldsymbol{x} \\
\boldsymbol{K}_{(0)}=\left[\begin{array}{lllll}
6.73 & 4.87 & 9.30 & 1.51 & -7.77
\end{array}\right] \\
\boldsymbol{K}_{(33)}=\left[\begin{array}{lllll}
14.8 & 6.36 & 20.1 & 3.48 & -17.2
\end{array}\right]
\end{aligned}
$$

それに対し，4. で提案した手法では $\bar{x}_{3}=0.652$ 程度まで 解が得られ，ディスクリプタ形式の柔軟性を利用することに よって保守性が軽減できた。なお，固有值指定条件付き非線 形 $\mathcal{H}_{\infty}$ 状態フィードバック $\mathcal{K}$ は以下のようになった.

$$
\begin{aligned}
\mathcal{K}: u & =\left(\boldsymbol{K}_{(0)}+x_{3}^{2} \boldsymbol{K}_{(33)}\right) \boldsymbol{x} \\
\boldsymbol{K}_{(0)} & =\left[\begin{array}{lllll}
7.96 & 5.41 & 11.0 & 1.80 & -9.14
\end{array}\right] \\
\boldsymbol{K}_{(33)} & =\left[\begin{array}{lllll}
8.26 & 3.76 & 12.7 & 2.24 & -9.53
\end{array}\right]
\end{aligned}
$$

Fig. 3 の実験結果からわかるように，ディスクリプ夕表現の 柔軟性を利用した 4. で提案した手法の方が $\bar{x}_{3}$ を大きく見 積もることができたため, 台車の目標值 $r^{r e f}$ が大きく変化 しても振動を抑えることができた.

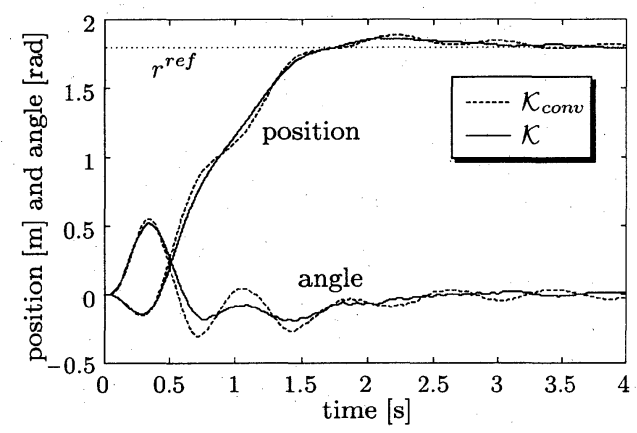

Fig. 3 experiment results $\left(W_{t}(p)=b_{t 0}+b_{t 1} p\right)$

\section{$5.3 W_{t}(p)=b_{t 0}+b_{t 1} p+b_{t 2} p^{2}\left(\left(\overline{\boldsymbol{C}}_{11} \neq \mathbf{o}\right)\right.$ の場合}

重み関数を $W_{t}(p)=(p+1.4)^{2} /\left(10 \times 1.4^{2}\right)$ とした。比較 のために文献 16) で提案した Hamilton-Jacobi 方程式の近 似解法を用いて非線形 $\mathcal{H}_{\infty}$ 状態フィードバック $\mathcal{K}_{a p p}$ を設 計すると，以下のようになった ${ }^{17)}$.

$$
\begin{aligned}
\mathcal{K}_{a p p}: u & =\left(\boldsymbol{K}_{(0)}+x_{3}^{2} \boldsymbol{K}_{(33)}\right) \boldsymbol{x} \\
\boldsymbol{K}_{(0)} & =\left[\begin{array}{lllll}
6.30 & 4.71 & 8.93 & 1.43 & -7.46
\end{array}\right] \\
\boldsymbol{K}_{(33)} & =\left[\begin{array}{lllll}
-4.94 & -2.04 & -0.220 & 0.526 & 6.50
\end{array}\right]
\end{aligned}
$$

また，4. で提案した手法では $\bar{x}_{3}=0.678$ 程度まで $(48)$ 式 の解が得られた。 その結果, 固有值指定条件付き非線形 $\mathcal{H}_{\infty}$ 状態フィードバック $\mathcal{K}$ は以下のようになった.

$$
\mathcal{K}: u=\left(\boldsymbol{K}_{(0)}+x_{3}^{2} \boldsymbol{K}_{(33)}\right) \boldsymbol{x}
$$

（注 1） $\boldsymbol{\Phi}(\boldsymbol{x}), \boldsymbol{Z}(\boldsymbol{x})$ は (18) 式の形式とした。また, sMI (19) 式の $\left(x_{3}^{2} / \bar{x}_{3}^{2}, x_{3}^{4} / \bar{x}_{3}^{4}, x_{4}^{2} / \bar{x}_{4}^{2}\right)$ を $(0,0,0),(1 / 4,0,0)$, $(3 / 4,1 / 2,0),(1,1,0),(0,0,1),(1 / 4,0,1),(3 / 4,1 / 2,1)$, $(1,1,1)$ で置き換えた 8 個の LMI を解いた ${ }^{12)}$.

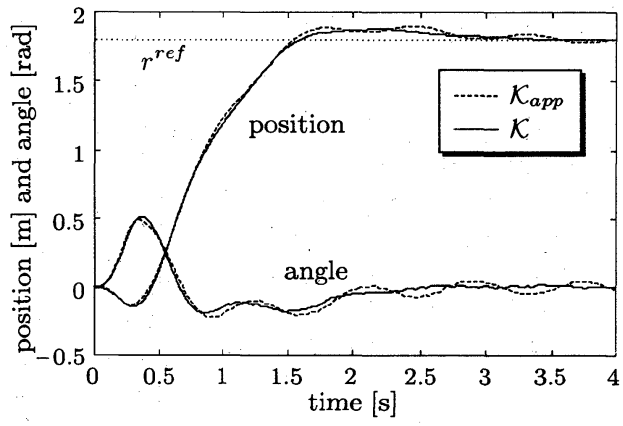

Fig. 4 experiment results $\left(\left(W_{t}(p)=b_{t 0}+b_{t 1} p+b_{t 2} p^{2}\right)\right.$

$$
\begin{aligned}
& \boldsymbol{K}_{(0)}=\left[\begin{array}{lllll}
6.38 & 4.79 & 9.62 & 1.57 & -7.40
\end{array}\right] \\
& \boldsymbol{K}_{(33)}=\left[\begin{array}{lllll}
7.54 & 3.69 & 11.9 & 2.11 & -8.81
\end{array}\right]
\end{aligned}
$$

Fig. 4 に示すように, 文献 16) の手法では Hamilton-Jacobi 方程式の近似解に基づいているために持続振動を生じたが， 4. で提案した手法では持続振動を生じていない。

\section{6.おわりに}

本論文では倒立振子システムを対象とし，ディスクリプタ 表現を利用した sLMI 条件に基づく固有值指定条件付き非線 形 $\mathcal{H}_{\infty}$ 状態フィードバックの設計法を提案した．特に 4. で はディスクリプタ表現の柔軟性を利用した場合,

(1) 非線形 $\mathcal{H}_{\infty}$ 制御問題における構造的不確かさの見 積もりに関する保守性を軽減できる

（2）円内への固有值指定問題の可解条件を等式制約のな い SLMI 条件で表すことができる

（3）非線形 $\mathcal{H}_{\infty}$ 制御問題，円内への固有值指定問題の 可解条件である SLMI を $\boldsymbol{x}$ に関する低次のべき級数で表 せ，また，解の形式を限定することによって 2 つの制御問 題を満足する保守性の少ない解が得られる

ことを示した．最後に，実機実験によって提案する設計法の 有効性を確認した。

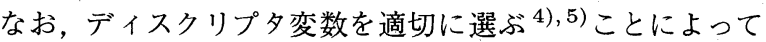
(21) 式の形式で表すことができるような非線形システムに対 しては，倒立振子システムの場合と同様，ディスクリプ夕表 現の柔軟性を利用した設計法が有効である考えられる。また， スケジューリングパラメータに依存したシステムの場合，定 理 5 の結果を極配置問題の可解条件として利用できる：今 後，他の実システムへの応用を検討していきたい.

最後に，本論文に対して貴重なご意見をいただいた査読者 の方々に感謝の意を表します.

\section{参 考文 献}

1）申，田村:ディスクリプタ型モデルに基づく $\mathcal{H}_{\infty}$ ロバスト準最 適制御, 計測自動制御学会論文集, 29-12, 1486/1488 (1993)

2）杉江，濱本：LMIによる 2 慣性共振系の制御，システム制御 情報学会論文誌, 9-5，219/226 (1996)

3）陳，杉江：パラメー夕依存リアプノフ関数に基づくディスクリ プタシステムのゲインスケジューリング，計測自動制御学会論 文集，34-9，1159/1163 (1998)

4）増淵，示村：ディスクリプタ形式に基づくゲインスケジュー! 
ング制御系設計，第 26 回制御理論シンポジウム資料， $21 / 24$ (1997)

5）増淵，示村：ゲインスケジューリング系の設計におけるディス クリプタ形式の利用について，システム制御情報学会論文誌， 12-7, 390/394 (1999)

6) 上里, 池田：ディスクリプタシステムに対する等式条件を含ま ない LMI 安定条件とそのロバスト安定化への応用, 計測自動 制御学会論文集，34-12，1854/1860 (1998)

7）陳，大西，藤中, 柴田：ディスクリプタ形式を利用したコス 卜保証制御，第 28 回制御理論シンポジウム資料，289/292 (1999)

8) I. Masubuchi, Y. Kamitane, A. Ohara and N. Suda: Control for Descriptor Systems: A Matrix Inequalities Approach, Automatica, 33-4, 669/673 (1997)

9）増淵, 小原, 須田：状態フィードバックによるディスクリプタ システムのロバスト安定化, 計測自動制御学会論文集, 30-12, 1553/1555 (1994)

10）渡辺, 内田, 藤田: 有限個の LMI 条件に基づいたスケジュー リングパラメータを持つ線形システムの解析, 第 24 回制御理 論シンポジウム資料，9/14 (1995)

11）内田, 渡辺：ゲインスケジューリング 一適応／非線形制御へ の展開, システム/制御／情報，42-6，306/311 (1998)

12）川田, 島津, 井上：ディスクリプタ形式に基づく非線形 $\mathcal{H}_{\infty}$ 制御とその倒立振子システムへの応用，第 21 回 Dynamical System Theory シンポジウム資料, 247/251 (1998)

13) A. J. van der Schaft: $\mathcal{L}_{2}$-gain Analysis of Nonlinear Systems and Nonlinear State Feedback $\mathcal{H}_{\infty}$ Control, IEEE Trans. Automat. Contr., AC-37-6, 770/784 (1992)

14) J. Imura, T. Sugie and T. Yoshikawa: A Hamilton-Jacobi Inequality Approach to the Strict $\mathcal{H}_{\infty}$ Control Problem of Nonlinear Systems, Automatica, 32-4, 645/650 (1996)

15）川田, 島津，井上:ディスクリプタ形式の表現能力を利用した 非線形 $\mathcal{H}_{\infty}$ 制御とその倒立振子システムへの応用, 第 28 回 制御理論シンポジウム資料, 257/262 (1999)

16）川田, 島津, 井上：Hamilton-Jacobi 方程式に基づく非線形 $\mathcal{H}_{\infty}$ 制御の近似実現, システム制御情報学会論文誌, 11-7, 401/410 (1998)

17）川田, 島津, 井上:倒立振子システムの非線形 $\mathcal{H}_{\infty}$ 状態フィード バック制御，計測自動制御学会論文集，35-2，294/296 (1999)

18）佐々木, 内田：拡張 2 次形式リアプノフ関数による非線形状 態フィードバックの構成法一線形行列不等式による凸解析ア プローチー，計測自動制御学会論文集，33-11，1111/1113 (1997)

\section{《付 録》}

\section{A. 定理 1 の証明}

紙面の関係上, 略証のみ示す. 文献 12) の定理 3 では $\boldsymbol{\Phi}(\boldsymbol{x})$ が定数行列 $\boldsymbol{\Phi}$ である場合について示されているが， $\boldsymbol{x}$ に依 存した $\boldsymbol{\Phi}(\boldsymbol{x})$ の場合, $\boldsymbol{\Phi}(\boldsymbol{x}) \boldsymbol{\Phi}^{-1}(\boldsymbol{x})=\boldsymbol{\Phi}^{-1}(\boldsymbol{x}) \boldsymbol{\Phi}(\boldsymbol{x})=\boldsymbol{I}$ よ り $\dot{\Phi}^{-1}(x)=-\Phi^{-1}(x) \dot{\Phi}(x) \Phi^{-1}(x)$ という関係式が成立す ることを利用すると, 文献 12) と同様に定理 1 を証明する ことができる。

\section{B. 定理 3 の証明}

$(26)$ 式における $\boldsymbol{E}^{\sharp}$ の構造を考慮すると, $\boldsymbol{E}^{\sharp} \boldsymbol{X}_{\mathcal{L}_{2}}^{T}(\boldsymbol{x})=$ $\boldsymbol{X}_{\mathcal{L}_{2}}(\boldsymbol{x}) \boldsymbol{E}^{\sharp T}$ となる

$$
\boldsymbol{X}_{\mathcal{L}_{2}}(\boldsymbol{x})=\left[\begin{array}{cc}
\boldsymbol{X}_{1}(\boldsymbol{x}) & \boldsymbol{X}_{\mathcal{L}_{2} 2}(\boldsymbol{x}) \\
\boldsymbol{X}_{\mathcal{L}_{2} 3}(\boldsymbol{x}) & \boldsymbol{X}_{\mathcal{L}_{2} 4}(\boldsymbol{x})
\end{array}\right]
$$

は $X_{1}(x)=X_{1}^{T}(x), X_{\mathcal{L}_{2} 3}(x)=O_{5 \times 5}$ でなければならず,
$\boldsymbol{X}_{\mathcal{L}_{2}}(\boldsymbol{x})$ は必ず $(29)$ 式の構造となる．このとき, $\boldsymbol{X}_{\mathcal{L}_{2}}(\boldsymbol{x})$ が正則であるための条件は

$$
\operatorname{det} \boldsymbol{X}_{\mathcal{L}_{2}}(\boldsymbol{x})=\operatorname{det} \boldsymbol{X}_{1}(\boldsymbol{x}) \operatorname{det} \boldsymbol{X}_{\mathcal{L}_{2} 4}(\boldsymbol{x}) \neq 0
$$

であるから，(27), (B.2) 式より $\boldsymbol{X}_{1}(\boldsymbol{x})=\boldsymbol{X}_{1}^{T}(\boldsymbol{x})>0$ で あり, $\boldsymbol{X}_{\mathcal{L}_{2} 4}(\boldsymbol{x})$ は正則である.

つぎに, $A_{c l}^{\sharp}(x):=A^{\sharp}(x)+B_{2}^{\sharp} K^{\sharp}(x)$ として Schur complement により (28) 式を書き換えると,

$$
\begin{array}{r}
\operatorname{He}\left[\boldsymbol{A}_{c l}^{\sharp}(\boldsymbol{x}) \boldsymbol{X}_{\mathcal{L}_{2}}^{T}(\boldsymbol{x})\right]-\boldsymbol{E}^{\sharp} \dot{\boldsymbol{X}}_{\mathcal{L}_{2}}^{T}(\boldsymbol{x})+\frac{1}{\gamma^{2}} \boldsymbol{B}_{1}^{\sharp} \boldsymbol{S}_{w} \boldsymbol{B}_{1}^{\sharp T} \\
+\boldsymbol{X}_{\mathcal{L}_{2}}(\boldsymbol{x}) \boldsymbol{C}_{1}^{\sharp T} \boldsymbol{S}_{z}^{-1} \boldsymbol{C}_{1}^{\sharp} \boldsymbol{X}_{\mathcal{L}_{2}}^{T}(\boldsymbol{x})<0
\end{array}
$$

となる。ただし, $(29)$ 式より $\boldsymbol{P}_{\mathcal{L}_{2}}(\boldsymbol{x}):=\boldsymbol{X}_{\mathcal{L}_{2}}^{-T}(\boldsymbol{x})$ は

$$
\begin{aligned}
& \boldsymbol{P}_{\mathcal{L}_{2}}(\boldsymbol{x})=\left[\begin{array}{cc}
\boldsymbol{P}_{1}(\boldsymbol{x}) & \boldsymbol{O}_{5 \times 5} \\
\boldsymbol{P}_{\mathcal{L}_{2} 3}(\boldsymbol{x}) & \boldsymbol{P}_{\mathcal{L}_{2} 4}(\boldsymbol{x})
\end{array}\right] \\
& \left\{\begin{array}{l}
\boldsymbol{P}_{1}(\boldsymbol{x}):=\boldsymbol{X}_{1}^{-1}(\boldsymbol{x}) \\
\boldsymbol{P}_{\mathcal{L}_{2} 3}(\boldsymbol{x}):=-\boldsymbol{X}_{\mathcal{L}_{2} 4}^{-T}(\boldsymbol{x}) \boldsymbol{X}_{\mathcal{L}_{2} 2}^{T}(\boldsymbol{x}) \boldsymbol{X}_{1}^{-1}(\boldsymbol{x}) \\
\boldsymbol{P}_{\mathcal{L}_{2} 4}(\boldsymbol{x}):=\boldsymbol{X}_{\mathcal{L}_{2} 4}^{-T}(\boldsymbol{x})
\end{array}\right.
\end{aligned}
$$

である。 また $\boldsymbol{X}_{1}(\boldsymbol{x}) \boldsymbol{X}_{1}^{-1}(\boldsymbol{x})=\boldsymbol{X}_{1}^{-1}(\boldsymbol{x}) \boldsymbol{X}_{1}(\boldsymbol{x})=\boldsymbol{I}$ より

$$
\dot{\boldsymbol{P}}_{1}(\boldsymbol{x})=\dot{\boldsymbol{X}}_{1}^{-1}(\boldsymbol{x})=-\boldsymbol{X}_{1}^{-1}(\boldsymbol{x}) \dot{\boldsymbol{X}}_{1}(\boldsymbol{x}) \boldsymbol{X}_{1}^{-1}(\boldsymbol{x})
$$

であるから，

$$
\boldsymbol{P}_{\mathcal{L}_{2}}^{T}(\boldsymbol{x}) \boldsymbol{E}^{\sharp} \dot{\boldsymbol{X}}_{\mathcal{L}_{2}}(\boldsymbol{x}) \boldsymbol{P}_{\mathcal{L}_{2}}^{T}(\boldsymbol{x})=-\dot{\boldsymbol{P}}_{\mathcal{L}_{2}}^{T}(\boldsymbol{x}) \boldsymbol{E}^{\sharp}
$$

となる.したがって, (B.3) 式の左から $\boldsymbol{P}_{\mathcal{L}_{2}}^{T}(\boldsymbol{x})$, 右から $\boldsymbol{P}_{\mathcal{L}_{2}}(\boldsymbol{x})$ をかけると

$$
\begin{aligned}
\operatorname{He}\left[\boldsymbol{P}_{\mathcal{L}_{2}}^{T}(\boldsymbol{x}) \boldsymbol{A}_{c l}^{\sharp}(\boldsymbol{x})\right]+\dot{\boldsymbol{P}}_{\mathcal{L}_{2}}^{T}(\boldsymbol{x}) \boldsymbol{E}^{\sharp}+\boldsymbol{C}_{1}^{\sharp T} \boldsymbol{S}_{z}^{-1} \boldsymbol{C}_{1}^{\sharp} \\
+\frac{1}{\gamma^{2}} \boldsymbol{P}_{\mathcal{L}_{2}}^{T}(\boldsymbol{x}) \boldsymbol{B}_{1}^{\sharp} \boldsymbol{S}_{w} \boldsymbol{B}_{1}^{\sharp T} \boldsymbol{P}_{\mathcal{L}_{2}}(\boldsymbol{x})<0 \text { (B. 5) }
\end{aligned}
$$

が得られる。いま，(B.5) 式を満足する $\boldsymbol{P}_{\mathcal{L}_{2}}(\boldsymbol{x})$ を用いて

$$
\begin{aligned}
\phi(x) & :=\boldsymbol{\xi}^{T} \boldsymbol{P}_{\mathcal{L}_{2}}^{T}(\boldsymbol{x}) \boldsymbol{E}^{\sharp} \boldsymbol{\xi}=\boldsymbol{\xi}^{T} \boldsymbol{E}^{\sharp T} \boldsymbol{P}_{\mathcal{L}_{2}}(\boldsymbol{x}) \boldsymbol{\xi} \\
& =\boldsymbol{x}^{T} \boldsymbol{P}_{1}(\boldsymbol{x}) \boldsymbol{x}>0(\boldsymbol{x} \neq \mathbf{o}, \boldsymbol{x} \in \mathcal{U})
\end{aligned}
$$

のように正定值関数 $\phi(x)$ を定義すると, その時間微分は

$$
\begin{aligned}
\dot{\phi}(\boldsymbol{x})= & \dot{\boldsymbol{\xi}}^{T} \boldsymbol{E}^{\sharp T} \boldsymbol{P}_{\mathcal{L}_{2}}(\boldsymbol{x}) \boldsymbol{\xi}+\boldsymbol{\xi}^{T} \boldsymbol{P}_{\mathcal{L}_{2}}^{T}(\boldsymbol{x}) \boldsymbol{E}^{\sharp} \dot{\boldsymbol{\xi}} \\
& +\boldsymbol{\xi}^{T} \dot{\boldsymbol{P}}_{\mathcal{L}_{2}}^{T}(\boldsymbol{x}) \boldsymbol{E}^{\sharp} \boldsymbol{\xi} \\
= & \boldsymbol{\xi}^{T} \operatorname{He}\left[\boldsymbol{P}_{\mathcal{L}_{2}}^{T}(\boldsymbol{x}) \boldsymbol{A}_{c l}^{\sharp}(\boldsymbol{x})\right] \boldsymbol{\xi} \\
& +\boldsymbol{w}^{\sharp T} \boldsymbol{S}_{w}^{1 / 2} \boldsymbol{B}_{1}^{\sharp T} \boldsymbol{P}_{\mathcal{L}_{2}}(\boldsymbol{x}) \boldsymbol{\xi} \\
& +\boldsymbol{\xi}^{T} \boldsymbol{P}_{\mathcal{L}_{2}}^{T}(\boldsymbol{x}) \boldsymbol{S}_{w}^{1 / 2} \boldsymbol{B}_{1}^{\sharp} \boldsymbol{w}^{\sharp} \\
< & -\gamma^{2}\left\|\boldsymbol{w}^{\sharp}-\frac{1}{\gamma^{2}} \boldsymbol{S}_{w}^{1 / 2} \boldsymbol{B}_{1}^{\sharp T} \boldsymbol{P}_{\mathcal{L}_{2}}(\boldsymbol{x}) \boldsymbol{\xi}\right\|^{2} \\
& +\gamma^{2}\left\|\boldsymbol{w}^{\sharp}\right\|^{2}-\left\|\boldsymbol{z}^{\sharp}\right\|^{2} \\
\leq & \gamma^{2}\left\|\boldsymbol{w}^{\sharp}\right\|^{2}-\left\|\boldsymbol{z}^{\sharp}\right\|^{2} \\
= & \gamma^{2}\|\bar{w}\|^{2}-\|\overline{\boldsymbol{z}}\|^{2} \\
& +\gamma^{2}\left(\boldsymbol{x}^{T} \boldsymbol{N}_{A}^{T} \boldsymbol{L}_{A}(\boldsymbol{x}) \boldsymbol{N}_{A} \boldsymbol{x}\right. \\
& \left.\quad+\dot{\boldsymbol{x}}^{T} \boldsymbol{N}_{E}^{T} \boldsymbol{L}_{E}(\boldsymbol{x}) \boldsymbol{N}_{E} \dot{\boldsymbol{x}}\right) \\
\boldsymbol{L}_{A}(\boldsymbol{x})= & \boldsymbol{\Sigma}_{A}^{T}(\boldsymbol{x}) \widetilde{\boldsymbol{S}}_{A}^{-1} \boldsymbol{\Sigma}_{A}(\boldsymbol{x})-\widetilde{\boldsymbol{S}}_{A}^{-1} \\
\boldsymbol{L}_{E}(\boldsymbol{x})= & \boldsymbol{\Sigma}_{E}^{T}(\boldsymbol{x}) \widetilde{\boldsymbol{S}}_{E}^{-1} \boldsymbol{\Sigma}_{E}(\boldsymbol{x})-\widetilde{\boldsymbol{S}}_{E}^{-1}
\end{aligned}
$$


となる.ここで, $(23)$ 式および $\widetilde{s}_{i}>0(i=1, \cdots, 4)$ より $\boldsymbol{L}_{A}(\boldsymbol{x}) \leq 0, \boldsymbol{L}_{E}(\boldsymbol{x}) \leq 0$ であるから，(B. 7$)$ 式は

$$
\dot{\phi}(\boldsymbol{x})<\gamma^{2}\|\bar{w}\|^{2}-\|\bar{z}\|^{2}
$$

となる。したがって, $\bar{w}=0$ のとき，

$$
\dot{\phi}(\boldsymbol{x})<-\|\overline{\boldsymbol{z}}\|^{2} \leq 0
$$

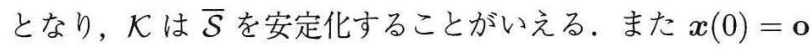
のとき, (B. 8) 式の両辺を $t=0$ から $\infty$ まで積分すると,

$$
\gamma^{2}\|\bar{w}\|_{2}^{2}-\|\bar{z}\|_{2}^{2} \geq 0
$$

となるから， $\mathcal{L}_{2}$ ゲイン条件 (9) 式を満足することがいえる. なお，(30), (B.4) 式より (31) 式は

$$
\begin{aligned}
\mathcal{K}: u= & \boldsymbol{Z}_{1}(\boldsymbol{x}) \boldsymbol{X}_{1}^{-1}(\boldsymbol{x}) \boldsymbol{x} \\
& -\boldsymbol{Z}_{2}(\boldsymbol{x}) \boldsymbol{X}_{\mathcal{L}_{2} 4}^{-T}(\boldsymbol{x}) \boldsymbol{X}_{\mathcal{L}_{2} 2}^{T}(\boldsymbol{x}) \boldsymbol{X}_{1}^{-1}(\boldsymbol{x}) \boldsymbol{x} \\
& +\boldsymbol{Z}_{2}(\boldsymbol{x}) \boldsymbol{X}_{\mathcal{L}_{2} 4}^{-T}(\boldsymbol{x}) \dot{\boldsymbol{x}}
\end{aligned}
$$

であるから，(30) 式おいて $Z_{2}(x)=O_{5}$ とし， $Z^{\sharp}(x)$ を (32) 式の構造に限定すると, 非線形状態フィードバック $\mathcal{K}$ は (33) 式のように $\boldsymbol{x}$ にのみ依存し, (22) 式の構造となる.

\section{C. 定理 4 の証明}

付録 $\mathbf{B}$ と同様, $\overline{\boldsymbol{E}}^{\sharp} \boldsymbol{X}_{e i g}^{T}(x)=\boldsymbol{X}_{e i g}(x) \overline{\boldsymbol{E}}^{\sharp T}$ となる正則 行列 $\boldsymbol{X}_{e i g}(\boldsymbol{x})$ は必ず (36) 式の構造となる。いま,

$$
\begin{aligned}
\overline{\boldsymbol{A}}_{c l}^{\sharp T}(\boldsymbol{x}) \boldsymbol{\psi} & =\lambda \overline{\boldsymbol{E}}^{\sharp T} \boldsymbol{\psi} \\
\overline{\boldsymbol{A}}_{c l}^{\sharp}(\boldsymbol{x}) & =\overline{\boldsymbol{A}}^{\sharp}(\boldsymbol{x})+\overline{\boldsymbol{B}}_{2}^{\sharp} \boldsymbol{K}^{\sharp}(\boldsymbol{x})
\end{aligned}
$$

を満足する $\psi \in \mathcal{C}^{10}\left(\mathcal{C}^{k}: k\right.$ 次元複素ベクトル)を

$$
\boldsymbol{\psi}=\left[\begin{array}{l}
\psi_{1} \\
\psi_{2}
\end{array}\right]\left(\psi_{1}, \psi_{2} \in \mathcal{C}^{5}\right)
$$

とおく.このとき, (35) 式の右から

$$
\Psi:=\left[\begin{array}{cc}
\psi & O_{10 \times 1} \\
\boldsymbol{O}_{10 \times 1} & \psi
\end{array}\right]
$$

をかけ, 左から $\boldsymbol{\Psi}$ の共役転置行列 $\boldsymbol{\Psi}^{*}$ をかけると，(C. 1) 式 および $\overline{\boldsymbol{E}}^{\sharp} \boldsymbol{X}_{\text {eig }}^{T}(\boldsymbol{x})=\boldsymbol{X}_{\text {eig }}(\boldsymbol{x}) \overline{\boldsymbol{E}}^{\sharp} よ り$

$$
\begin{gathered}
\boldsymbol{Q}(\lambda) \boldsymbol{\psi}^{*} \overline{\boldsymbol{E}}^{\sharp} \boldsymbol{X}_{\text {eig }}^{T}(\boldsymbol{x}) \boldsymbol{\psi} \geq 0 \\
\boldsymbol{Q}(\lambda):=\left[\begin{array}{cc}
q\left(\lambda+\lambda^{*}\right)+d & \lambda^{*} \\
\lambda & 1
\end{array}\right], d=r^{2}-q^{2}
\end{gathered}
$$

となる。ただし， $\lambda^{*}$ は $\lambda$ の共役複素数である。ここで， $\boldsymbol{X}_{\text {eig1 } 1}(\boldsymbol{x})=\boldsymbol{X}_{\text {eig1 }}^{T}(x)>0$ より

$$
\begin{aligned}
\boldsymbol{\psi}^{*} \overline{\boldsymbol{E}}^{\sharp} \boldsymbol{X}_{\text {eig }}^{T}(\boldsymbol{x}) \boldsymbol{\psi} & =\boldsymbol{\psi}^{*} \boldsymbol{X}_{\text {eig }}(\boldsymbol{x}) \overline{\boldsymbol{E}}^{\sharp T} \boldsymbol{\psi} \\
& =\boldsymbol{\psi}_{1}^{*} \boldsymbol{X}_{\text {eig } 1}(\boldsymbol{x}) \boldsymbol{\psi}_{1}>0
\end{aligned}
$$

であるから，(C.3) 式より $Q(\lambda) \geq 0$ であることがいえ，

$$
(\alpha-q)^{2}+\beta^{2} \leq r^{2}
$$

という関係式が得られる。したがって, 非線形状態フィード バック (38) 式は特性方程式 (39) 式の根 $\lambda=\alpha+j \beta$ を中心 $(q, 0)$, 半径 $r$ の円内に配置する。

なお, $Z_{e i g}^{\sharp}(x)$ の構造を特に (37) 式と限定すれば, 付録 B
と同様, 非線形状態フィードバック (38) 式は (41) 式のよう に $\boldsymbol{x}$ にのみ依存し, (22) 式の構造となる.また, (C.1) 式は

$$
\overline{\boldsymbol{A}}_{c l}^{T}(\boldsymbol{x}) \boldsymbol{\psi}_{2}=\lambda \boldsymbol{\psi}_{1}, \boldsymbol{\psi}_{1}=\overline{\boldsymbol{E}}^{T}(\boldsymbol{x}) \boldsymbol{\psi}_{2}
$$

のように分割できる。ただし, $\overline{\boldsymbol{A}}_{c l}(\boldsymbol{x}):=\overline{\boldsymbol{A}}(\boldsymbol{x})+\overline{\boldsymbol{B}}_{2} \boldsymbol{K}(\boldsymbol{x})$ である。したがって，(C.1) 式は元のシステム (11) 式に対 する特性方程式 (12) 式に相当する

$$
\overline{\boldsymbol{A}}_{c l}^{T}(x) \boldsymbol{\psi}_{2}=\lambda \overline{\boldsymbol{E}}^{T}(x) \boldsymbol{\psi}_{2}
$$

と等価であり, 非線形状態フィードバック (41) 式が特性方 程式 (12) 式の根 $\lambda=\alpha+j \beta$ を中心 $(q, 0)$, 半径 $r$ の円内 に配置することがいえる。

\section{D. 定理 5 の証明}

$\boldsymbol{K}^{\sharp}(\boldsymbol{x})=\left[\begin{array}{ll}\boldsymbol{X}_{1}^{-1}(\boldsymbol{x}) \boldsymbol{Z}_{1}(\boldsymbol{x}) & \boldsymbol{O}_{1 \times 5}\end{array}\right]$ として, (C. 1) 式 を満足する $\psi$ を $\psi_{1}$ と $\psi_{2}$ に分割すると, 付録 $\mathbf{C}$ と同様, (C. 7) 式が得られ，(C.1) 式が元のシステム (11) 式に対す る特性方程式 (12) 式に相当する (C. 7) 式と等価であること がわかる、いま，(42) 式の右から

$$
\widehat{\boldsymbol{\Psi}}:=\left[\begin{array}{cc}
\psi & O_{10 \times 1} \\
\boldsymbol{O}_{5 \times 1} & \psi_{1}
\end{array}\right]
$$

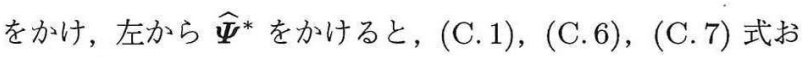
よび $\overline{\boldsymbol{E}}^{\sharp} \boldsymbol{X}_{\text {eig }}^{T}(\boldsymbol{x})=\boldsymbol{X}_{\text {eig }}(\boldsymbol{x}) \overline{\boldsymbol{E}}^{\sharp}$ より

$$
Q(\lambda) \psi_{1}^{*} X_{1}(x) \psi_{1}>0
$$

となる.ここで, $\boldsymbol{X}_{1}(\boldsymbol{x})=\boldsymbol{X}_{1}^{T}(\boldsymbol{x})>0$ であるから，(D.2) 式 より $\boldsymbol{Q}(\lambda)>0$ であり, 以下の関係式が得られる.

$$
(\alpha-q)^{2}+\beta^{2}<r^{2}
$$

したがって, 非線形状態フィードバック (33) 式が特性方程 式 (12) 式の根 $\lambda=\alpha+j \beta$ を中心 $(q, 0)$, 半径 $r$ の円内に 配置することとがいえる。

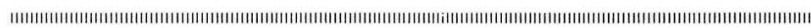

\section{[著 者 紹 介]}

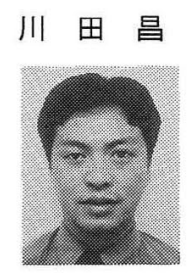

克 (正会員)

1997 年 3 月立命館大学大学院理工学研究科博 士後期課程修了. 同年 4 月より同大学理工学部助 手 (任期制) を経て, 98 年 4 月より舞鶴工業高等 専門学校電子制御工学科助手. 非線形システムの ロバスト制御とその応用に関する研究に従事. 博 士 (工学). システム制御情報学会の会員.

井上和夫 (正会員)

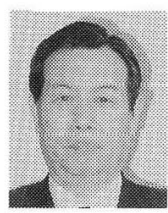

1966 年 3 月大阪大学大学院工学研究科博士課 程修了. 同年 4 月より立命館大学理工学部講師, 助教授を経て, 71 年より教授. ヒューマンインタ フェース, 適応制御などに関する研究に従事. 工 学博士. システム制御情報学会, 電気学会, 電子 情報通信学会, IEEE などの会員. 